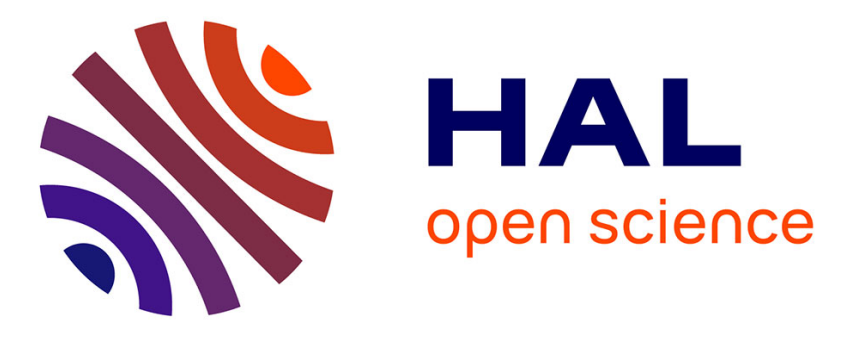

\title{
Les stèles gravées néolithiques de Beyssan à Gargas (Vaucluse)
}

André d'Anna, Christiane Bosansky, Ludovic Bellot-Gurlet, François-Xavier Le Bourdonnec, Jean-Louis Guendon, Adrien Reggio, Stéphane Renault

\section{- To cite this version:}

André d'Anna, Christiane Bosansky, Ludovic Bellot-Gurlet, François-Xavier Le Bourdonnec, JeanLouis Guendon, et al.. Les stèles gravées néolithiques de Beyssan à Gargas (Vaucluse). Bulletin de la Société préhistorique française, 2015, 112 (4), pp.761-768. 10.3406/bspf.2015.14599 . hal-01449407

\author{
HAL Id: hal-01449407 \\ https://hal.science/hal-01449407
}

Submitted on 29 Jul 2018

HAL is a multi-disciplinary open access archive for the deposit and dissemination of scientific research documents, whether they are published or not. The documents may come from teaching and research institutions in France or abroad, or from public or private research centers.
L'archive ouverte pluridisciplinaire HAL, est destinée au dépôt et à la diffusion de documents scientifiques de niveau recherche, publiés ou non, émanant des établissements d'enseignement et de recherche français ou étrangers, des laboratoires publics ou privés. 


\title{
Les stèles gravées néolithiques de Beyssan à Gargas (Vaucluse)
}

\author{
André D’Anna, Christiane Bosansky, Ludovic Bellot-Gurlet, \\ François-Xavier Le Bourdonnec, Jean-Louis Guendon, Adrien Reggio et Stéphane Renault
}

Résumé : Les stèles provençales néolithiques gravées sont bien connues avec plus de cinquante exemplaires. Les deux stèles de Gargas ont été découvertes à l'occasion d'une prospection de surface sur les marges nord de la plaine du Calavon à environ $45 \mathrm{~km}$ à l'est d'Avignon. C'est là une région de grande densité de gisements néolithiques dont plusieurs ont fait l'objet de fouilles : la Brémonde à Buoux, les Martins à Roussillon, les Fabrys à Bonnieux et le dolmen de l'Ubac à Goult.

La stèle 1 est entière mais localement altérée et sa surface est encroûtée. On peut cependant reconnaître un calcaire dense et fin. La forme est triangulaire. Les flancs et le sommet sont faiblement convexes. La face est légèrement concave et témoigne d'un travail de dressage avant la réalisation du décor. Au centre, la partie réservée n'est pas en creux comme c'est généralement le cas sur les stèles provençales. Elle est détériorée. Cette zone réservée verticale, est resserrée à mi-hauteur puis s'évase vers le bas. Les détails anatomiques, bloc sourcils-nez-yeux, ne sont pas visibles du fait de la détérioration, mais ce qui reste de la zone ne montre ni relief ni creux. De part et d'autre deux bandes margées verticales sont gravées de hachures obliques simples. À la partie supérieure, le décor est constitué de quatre registres : deux bandes horizontales lisses, une bande de chevrons simples horizontaux, deux bandes horizontales de hachures obliques de droite à gauche et une bande de chevrons doubles horizontaux. Le décor gravé est rehaussé de pigment rouge vif déterminé comme du cinabre.

La stèle 2 n'est pas totalement intègre, la base est absente. L'ensemble de la surface est encroûté. Le matériau utilisé est un peu plus grenu que celui de la stèle 1. La surface de la face, plate à localement convexe, ne semble pas avoir été dressée. Le décor est gravé, rehaussé de couleur rouge vif, également du cinabre. Il est moins soigné que celui de la stèle 1. Il est organisé en trois zones autour de la surface réservée verticale qui ne porte ni creux, ni relief sculptés, ni apparemment de couleur. Les attributs anatomiques, bloc sourcils-nez-yeux, ne sont pas représentés. Les bandes verticales latérales sont constituées de hachures obliques. À la partie supérieure on observe deux bandes horizontales : une bande étroite dont le décor est difficilement lisible et au-dessus une frise de chevrons. La structure globale de ce décor est conforme à celle observée sur les autres stèles provençales, mais la bande centrale est originale par sa forme et l'absence de figuration.

La localisation de la découverte, au nord du Luberon, dans la partie nord de l'aire de répartition des stèles provençales, confirme que plusieurs sont liées à la vallée du Calavon, comme d'autres le sont à celles de l'Arc et de la Durance.

Si la structure du décor des stèles de Gargas est conforme à ce qui était connu, dans le détail il y a des aspects originaux : la forme du visage est à bords évasés vers le bas et ne fait apparaître ni le nez, ni les yeux.

Les stèles de Gargas ont été recueillies en surface et le mobilier associé indique qu'il y avait là un contexte, probablement mal conservé du fait de la localisation du site et des labours successifs. Un premier examen montre une très grande homogénéité. L'industrie en silex taillé est très majoritairement sur silex « blond » de la région de Murs. La station a également livré des pièces en obsidienne du Monte Arci en Sardaigne.

Bien que généralement privées de contexte les stèles provençales gravées sont bien datées. Elles ont toujours été attribuées au Chasséen récent. Elles seraient donc antérieures aux manifestations de la grande statuaire de la fin du Néolithique. Les découvertes de Gargas semblent le confirmer; elles s'inscrivent dans un cadre chronoculturel de la fin du Néolithique moyen entre 3800 et 3600 av. J.-C.

Si la datation des stèles provençales semble établie, il n'en n'est pas de même pour leur contexte précis et leur fonction. Un lien avec des faits funéraires peut-être évoqué, mais les stèles ne sont pas directement associées aux sépultures. Par ailleurs, aucune des grandes nécropoles à sépultures en fosses de la fin du Néolithique moyen ne livre de tels monuments. Il semblerait donc que les stèles provençales constituent un phénomène qui pourrait être associé à des locus particuliers d'établissements plus vastes comme cela est le cas apparemment à Beyssan.

Mots-clefs : Néolithique moyen, Provence, stèles gravées.

Abstract: The Provence Neolithic stelae with chevron ornamentation are well known, with more than fifty monuments currently recorded. The two new stelae from Gargas were discovered during a field survey on the northern 
border of the Calavon plain, about $45 \mathrm{~km}$ east of Avignon. The area has a high concentration of archaeological sites, many excavated in the past: La Brémonde (Buoux), Les Martin (Roussillon), Les Fabrys (Bonnieux) and the Ubac dolmen (Goult).

Stela 1 is complete but deteriorated in places. Its surface is encrusted. It is however possible to identify the stone as a compact, fine limestone. The general shape is triangular, with the sides and top slightly convex. The slightly concave front face indicates that the surface was pick-dressed prior to being decorated. The reserved part at the centre is deteriorated. It is not hollowed as it is usually the case for Provence stelae. This reserved vertical area narrows in its middle then gets wider towards the bottom. Anatomical details such as the eyebrows-nose-eyes block are not visible because of the deterioration of the stela but what is left of the reserved area does not show any relief or hollows. Two vertical bands decorated with parallel oblique lines running outwards are engraved on both sides of this area. The ornamentation on the top part consists of four levels: two smooth horizontal bands, one row of simple horizontal chevrons, two horizontal series of parallel oblique lines, and one row of double horizontal chevrons. The engravings are enhanced with a bright red colour which has been identified as cinnabar.

Stela 2 is not complete: its base is missing. Its whole surface is encrusted. The raw material is a little more grainy than the one used for stela 1 . The flat, locally convex, main face does not seem to have been pick-dressed. The ornamentation is engraved and also enhanced with a bright red colour here again identified as cinnabar. It is simpler and less carefully made than on stela 1. It is organized in three areas around the vertical reserved surface, which does not seem to have hollows, sculpted reliefs or colour. No anatomical details (eyebrows-nose-eyes) are represented. The lateral vertical bands are simple, consisting of series of parallel oblique lines running outwards. Two horizontal bands appear on the top part of the stela: a narrow band, whose ornamentation is difficult to see, above which is a series of double chevrons. The overall structure of this artwork is in accordance with that seen on other stelae in Provence, but the central band, with its form and lack of any realist figuration, is a more original feature.

The site of the discovery, on the Calavon plain (North Luberon), is in the northern part of the distribution area of engraved stelae. This confirms that several stelae are associated with the Calavon valley, in the same way as other stelae groups are associated with the Arc or Durance valleys. The two Gargas stelae are part of a group initially called B, then 'Lower-Durance group' or 'chevron-ornamented stelae group or Durance-group'. The ornamentation on these stelae is limited to the representation of a head consisting of the shape of the face, hollowed out, and a series of chevrons carved in relief. The ornamentation on the Gargas stelae is not in relief but engraved. Following Gagnière and Granier's definition a third group (stelae with painted ornamentation, or Château Blanc type) was identified, including the stelae from Château Blanc (Ventabren) and those from Goult/Ubac. These were carefully shaped, were not engraved but have traces of red pigment. While the overall structure of the engraved ornamentation of the Gargas stelae is consistent with what was already known, some details are more original: the shape of the face has sides widening towards the bottom and does not include a nose or eyes.

The two stelae from Gargas were unearthed subsequently to ploughing and associated material points to evidence for a context which is probably poorly preserved due to the location of the site and regular agricultural work. This material is a particularly spectacular assemblage as is sometimes found in Vaucluse. It comes from various locations around Beyssan. A first examination shows significant homogeneity. Chipped flint tools dominate the assemblage, with a majority of "blond" flint from the area of Murs. Besides flint, the site has yielded finds in obsidian originating from Monte Arci, Sardinia.

Though generally found outside any archaeological context, Provence stelae with chevron ornamentation are relatively well dated. They have always been dated to the Middle Neolithic, and more exactly to the Late Chasséen. They therefore precede the tradition of large anthropomorphic statuary of the end of the Neolithic period. The Gargas discoveries confirm this; their chrono-cultural context dates to the end of the Middle Neolithic, between 3800 and 3600 BC.

While the Provence stelae can be considered as well dated overall, the nature of their context has not yet been well understood. A link with funerary practices is obvious at Goult and Château Blanc and can be suggested at Bastidonne and maybe at Puyvert. However, stelae are not directly associated with burials. Moreover, late Neolithic cemeteries which have been extensively excavated have not yielded such monuments. It seems therefore that the Provence stelae are a particular phenomenon, which could be associated with sites of an 'intermediary' type, which could have been specific places associated with larger settlements as seems to be the case at Beyssan [translation Guillaume Robin].

Key-words: Middle Neolithic, Provence, engraved stelae.

$\mathrm{L}$ ES STÈLES provençales néolithiques à décor de chevrons sont des petits monuments bien connus. Régulièrement de nouvelles découvertes viennent compléter les inventaires et les données iconographiques. Ces stèles, une cinquantaine actuellement inventoriée, restent le plus souvent hors contexte ou en situation imprécise ou mal perçue. Les deux nouvelles stèles trouvées à Gargas ne dérogent pas à la règle puisqu'elles ont été recueillies en surface. Cependant les informations relatives au site auquel elles étaient associées sont ici relativement riches, précises et homogènes. Elles permettent de confirmer les hypothèses déjà proposées d'une datation de la fin du Néolithique moyen. Cet article a pour objectif de faire connaître cette découverte avec la description des deux stèles et de souligner les nouveaux éléments qu'elles apportent à la réflexion et à la discussion sur le sujet ${ }^{(1)}$. 


\section{SITUATION ET CIRCONSTANCES}

$\mathrm{D}$ epuis plusieurs années l'une de nous (C. B.) poursuit des ramassages de surface sur une très vaste " station » néolithique connue depuis longtemps. Ces découvertes ont été effectuées, entre les monts de Vaucluse et le Luberon, à la marge nord du bassin d'Apt et de la plaine du Calavon-Coulon, un peu en aval d'Apt, sur plusieurs parcelles des communes de Gargas, Apt et Saint-Saturninlès-Apt (Vaucluse). Elles sont localisées autour de la « campagne Beyssan » (la maison Santi), Beïssan sur la carte IGN au 1/25000 (3142 est, Apt Ouest Bonnieux), située à environ $45 \mathrm{~km}$ à l'est d'Avignon, 3,5 km au nordouest d'Apt et 1,3 km à l'est de Gargas (fig. 1).

Les espaces prospectés s'étendent sur plusieurs hectares sur le versant sud-est de la colline de Perréal-Sainte Radegonde (468-471 m), entre les ruisseaux de la Riaille et des Grandes Terres qui descendent vers le sud et le Calavon (fig. 2). La carte géologique au 1/50 000 indique que l'on est là sur des éboulis et épandages recouvrant des marnes du Gargasien, dominés par des sables et grès verts du Clansayen, des argiles de l'Éocène supérieur (Ludien) et des calcaires, argiles et marnes de l'Oligocène (Stampien, Sannoisien) de Sainte-Radegonde Perréal qui couronnent ici la série (Goguel, 1966). La morphologie actuelle résulte de la succession des travaux agricoles mais montre nettement l'alternance de petits thalwegs et de leurs interfluves principalement constituées de terrains tendres et donc largement soumis à l'érosion.

La vallée du Calavon, entre le Luberon au sud et les monts de Vaucluse et le plateau d'Albion au nord, est une région de grande densité de sites archéologiques, en particulier de «stations néolithiques de surface », connues de longue date (Pottier, 1878; Cotte, 1924), telles, dans ce secteur, la Bladayre à $3 \mathrm{~km}$ à l'ouest de Beyssan (Courtin, 1974 , fig. 53 , p. 111, p. 132) ou encore la Verrière à Goult (Marchesi, 1990). Un peu en marge de la vallée, il convient également de mentionner, vers le nord, les grottes de la Lave et de Valoubeau à Saint-Saturnin-lès-Apt

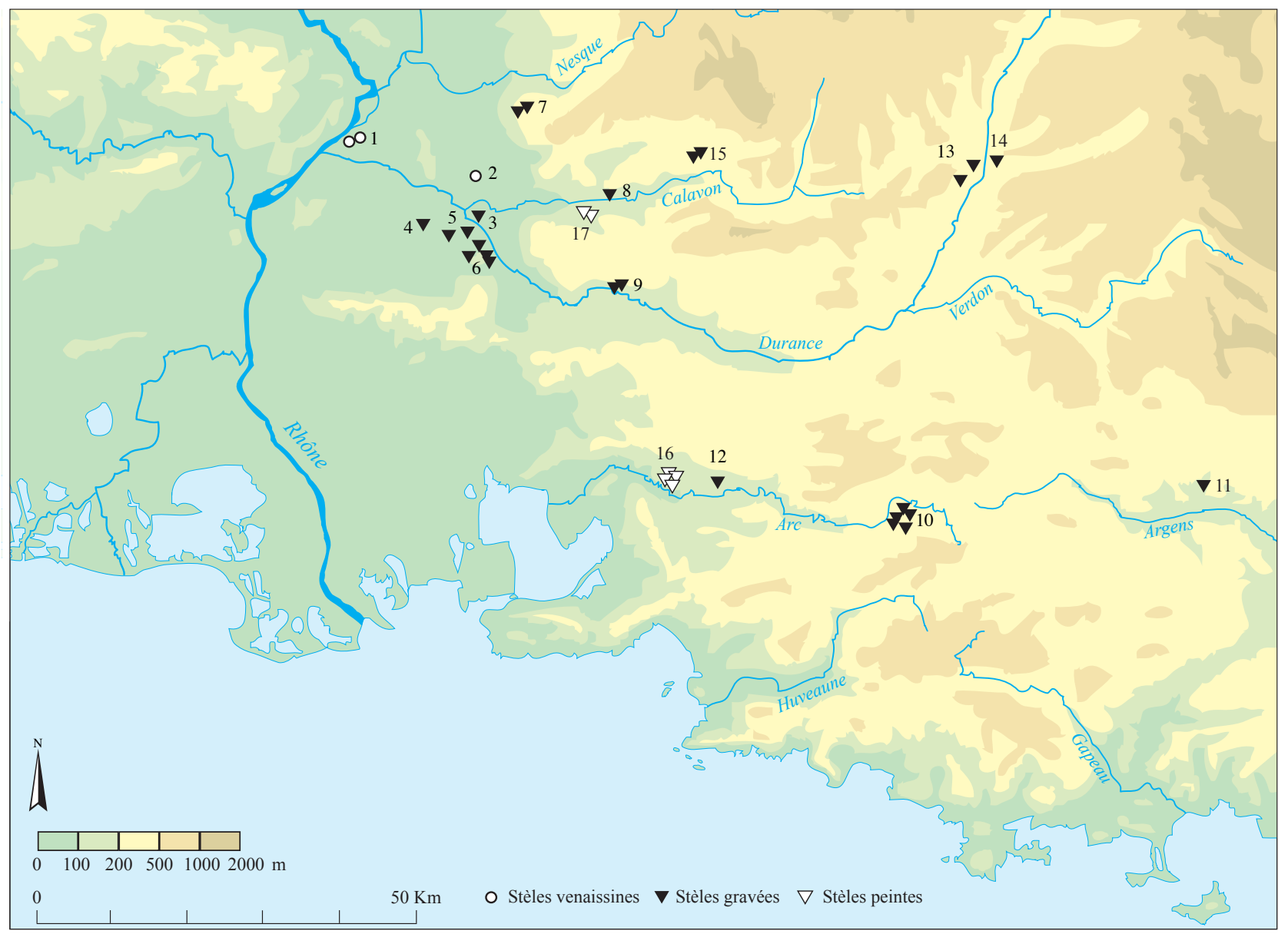

Fig. 1 - Carte de localisation du site de Beyssan à Gargas, Vaucluse et répartition des stèles anthropomorphes en Provence. 1 : Avignon, la Balance et Avignon, Rocher des Doms; 2 : Isles-sur-La-Sorgue, la Bastide; 3 : Cavaillon; 4 : Eygalières, Vallongues; 5 : Orgon, Font de Malte et Mont Sauvy; 6 :Sénas, la Puagère 1 à $8 ; 7:$ Le Beaucet 1 et 2; $8:$ Goult, Ponty sud; 9 : Puyvert, la Lombarde 1 à 4 ; 10 : Trets, la Bastidonne, 1 à 18; 11 : Villecroze, le Frigouret; 12 :Aix-en-Provence, Sextius-Mirabeau; 13 : Villeneuve, les Petites Lombardes; 14 : Oraison, la Grande Bastide; 15 : Gargas, Beyssan; 16 : Ventabren, Château Blanc 1 à 7 ; 17 : Goult, 1'Ubac 1 et 2.

Fig. 1 - Map showing the location of the site of Beyssan, Gargas, Vaucluse (no.15) and the distribution of anthropomorphic stelae in Provence. 


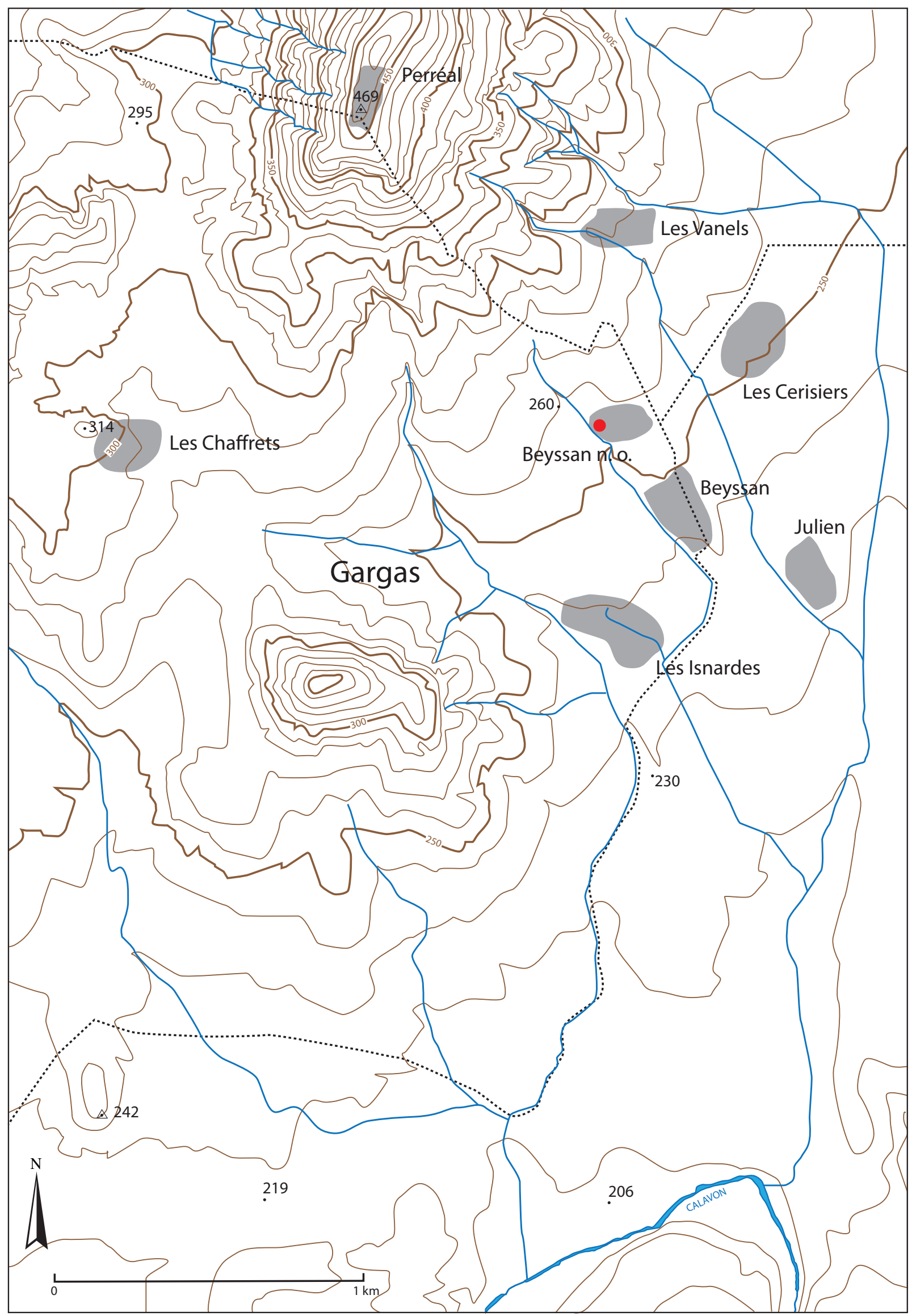

Fig. 2 - Gargas, localisation de la découverte des deux stèles anthropomorphes et des « stations » de Beyssan.

Fig. 2 - Gargas, location of the place of discovery of the two anthropomorphic stelae and of the Beyssan 'surface stations'. 
(Courtin, 1974; Sauzade, 1983) à environ 6 km au nordouest de Beyssan et vers le sud, dominant la vallée, les stations du plateau des Claparèdes et du Luberon central, à environ 6-7 km au sud de Beyssan (Arnaud d'Agnel, 1901; Sautel et al., 1931).

Plusieurs sites importants dans la vallée elle-même ou ses marges ont fait l'objet de sondages et de fouilles : les abris de Roquefure et du Bois Sauvage à $6 \mathrm{~km}$ au sud-ouest (Brochier et Livache, 2004), la Brémonde à Buoux à environ $8 \mathrm{~km}$ au sud - sud-est (D'Anna et al., 1989; D'Anna, 1993), les Martins à Roussillon à un peu plus de $8 \mathrm{~km}$ à l'ouest (D'Anna, 1993), les Fabrys à Bonnieux à environ $10 \mathrm{~km}$ au sud-ouest (Bretagne et D'Anna, 1988), la Beaume Croupatière (berceau de la Société préhistorique française) également à Bonnieux à environ $11 \mathrm{~km}$ au sudouest (Sauzade, 1983) et le dolmen de l'Ubac à Goult à $12,5 \mathrm{~km}$ au sud-ouest (Sauzade et al., 2003; Bizot et al., 2004). Enfin, les abris à peintures de Saint-Saturninlès-Apt, Beaume Peinte et Font-Jouval (Hameau, 1998, 2004) sont à environ $7 \mathrm{~km}$ au nord-ouest de Beyssan, les abris de Beaume Brune à Gordes et Joucas (Hameau, 1999) sont un peu plus loin, à environ $11 \mathrm{~km}$ au nord - nord-ouest.

Dans ce contexte, à la suite des labours du printemps 2014, deux stèles anthropomorphes ont été découvertes et identifiées par l'une de nous (C. B.). Elles proviennent d'une parcelle entre les campagnes Beyssan et les Vanels au nord, à environ $300 \mathrm{~m}$ au nord-ouest de la maison Santi (campagne Beyssan); l'espace est en pente vers l'ouest, sur la rive gauche du petit Vallat de Beyssan à une trentaine de mètres du fond du talweg. Dans l'état actuel des données il semble que le lieu de découverte puisse être localisé à la limite nord-ouest d'un vaste établissement connu comme la station des Isnardes (fig. 2). Il semble constitué de différents locus dont témoigne la dispersion des ramassages. La très grande abondance du mobilier en surface suggère que le site a été largement altéré et partiellement détruit par les labours et l'érosion naturelle.

\section{LES DEUX STÈLES}

\section{La stèle 1}

La stèle 1 est entière, mais localement altérée; sa hauteur totale est de $29 \mathrm{~cm}$, sa largeur maximale au sommet de $20,5 \mathrm{~cm}$, l'épaisseur de la partie supérieure de $7,40 \mathrm{~cm}$ et l'épaisseur de la partie inférieure de $6 \mathrm{~cm}$ (fig. 3, 4, 5 et 6).

L'ensemble de la surface est très encroûté. On peut cependant reconnaître une plaquette d'un calcaire relativement dense et très fin, sublithographique, très homogène, de couleur beige, à cassure conchoïdale. Il montre des variations latérales de faciès. Il s'agit probablement d'un calcaire oligocène tel qu'il en existe à la partie supérieure du massif de Sainte Radegonde Péréal au nord immédiat de Beyssan et surtout à une dizaine de kilomètres au nord-ouest dans le bassin (fossé) de Murs.

La forme générale de la stèle est triangulaire, la base est « pointue », les flancs et le sommet légèrement convexes. La morphologie de la base laisse supposer que la stèle devait être plantée de 6 à $9 \mathrm{~cm}$. L'analyse technologique précise à partir des stigmates de fabrication est impossible à réaliser du fait de l'importance de l'encroûtement. On peut cependant remarquer que la première phase de la mise en forme correspond à des enlèvements d'éclats sur les faces dorsales et latérales. La face dorsale est brute (fig. 5a). La partie supérieure a fait l'objet d'un façonnage soigné par raclage dont les stries parallèles sont localement visibles (fig. 5b). La face principale montre une surface légèrement concave qui témoigne d'un travail de dressage préalable à la réalisation du décor (fig. 5c). Cette face a été largement endommagée postérieurement à la décoration en particulier au centre de la partie supérieure, par des épaufrures peu épaisses (1,4 mm max.) qui laissent voir le vestige du fond des gravures. Au centre, la partie réservée n'est pas en creux comme c'est généralement le cas sur les autres stèles provençales et il n'y a pas de véritable relief. Là où elles sont bien lisibles les gravures sont larges de 0,2 à $0,4 \mathrm{~mm}$ et profondes de 0,5 à $0,7 \mathrm{~mm}$, rarement jusqu'à $1 \mathrm{~mm}$. L'ensemble peut être restitué entièrement (fig. 6). Il est organisé en trois zones autour de la surface réservée qui ne porte ni creux, ni relief sculpté, ni apparemment de couleur. La zone réservée, verticale, large de $8 \mathrm{~cm}$ au maximum, est légèrement resserrée à mi-hauteur $(6 \mathrm{~cm})$ puis s'évase vers le bas jusqu'à occuper toute la largeur de la stèle. Les détails anatomiques ne sont pas visibles du fait de la détérioration, mais ce qui reste de la zone réservée ne montre ni relief ni creux ce qui suggère que le bloc sourcils-nez-yeux n'était pas représenté, ni en relief ni peint (voir ci-dessous). Cette forme de visage, évasée vers le bas, n'existait pas dans la typologie antérieure (D'Anna et al., 2004, fig. 5, p. 14), et il convient donc de la rajouter pour constituer un type 7 (ce type existe également sur d'autres stèles inédites, voir ci-après).

De part et d'autre de la zone réservée, se trouvent deux bandes margées verticales décorées de hachures obliques simples descendantes de l'intérieur vers l'extérieur; les deux bandes sont donc d'orientation opposée. Il n'y a pas de chevrons. Les deux bandes sont composées de deux séries verticales de hachures (B2) de même orientation mais d'inégale largeur (la nomenclature de l'organisation des chevrons et hachures est celle figurée dans D'Anna et al., 2004, fig. 6, p. 15). Les séries intérieures font $1,80 \mathrm{~cm}$ à droite et $1,90 \mathrm{~cm}$ à gauche. Les séries extérieures en bordure de motifs sont plus larges ( 2,7 à $2,8 \mathrm{~cm}$ à droite et non mesurables à gauche, mais identiques) et limitées par une verticale (autant que l'on puisse en juger). À gauche la bande est moins haute $(13,5 \mathrm{~cm})$; à droite la bande descend plus bas $(14 \mathrm{~cm}$ de haut). L'aspect général est relativement régulier et soigné, bien que l'oblicité ne soit pas toujours régulière en particulier à droite avec une bande qui paraît un peu moins soignée; la largeur des plats est faiblement irrégulière de 0,30 à $0,40 \mathrm{~cm}$ à gauche et de 0,40 à $0,50 \mathrm{~cm}$.

À la partie supérieure le décor est complexe et moins facilement lisible du fait de l'encroûtement et des altérations. Il est constitué de quatre registres horizontaux. 


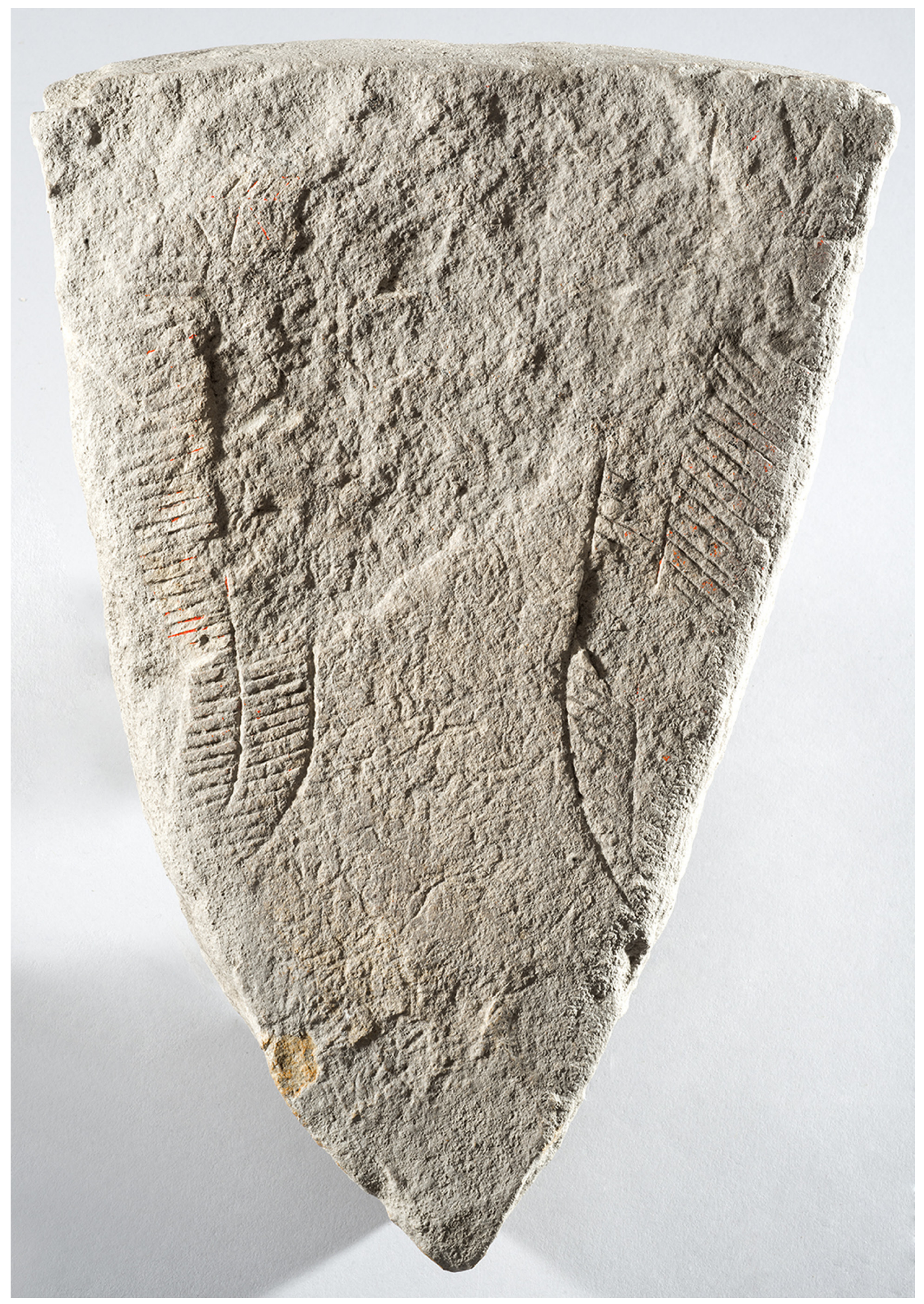

Fig. 3 - Gargas, stèle 1 de Beyssan (cliché C. Durand, AMU-CNRS-CCJ).

Fig. 3 - Gargas, stela 1 from Beyssan (photo C. Durand, AMU-CNRS-CCJ). 


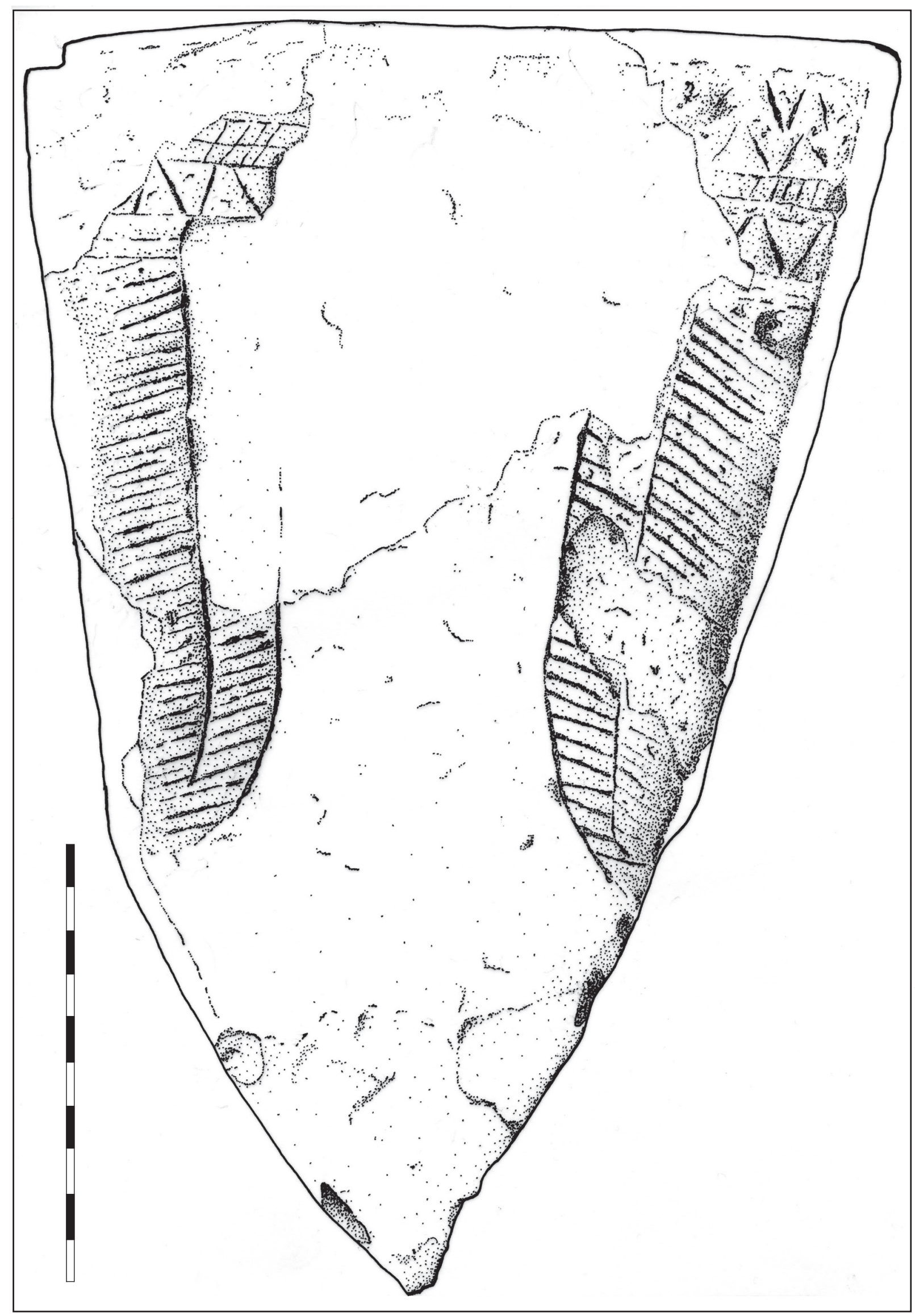

Fig. 4 - Gargas, stèle 1 de Beyssan (relevé et dessin A. D’Anna).

Fig. 4 - Gargas, stela 1 from Beyssan (drawing A. D'Anna). 


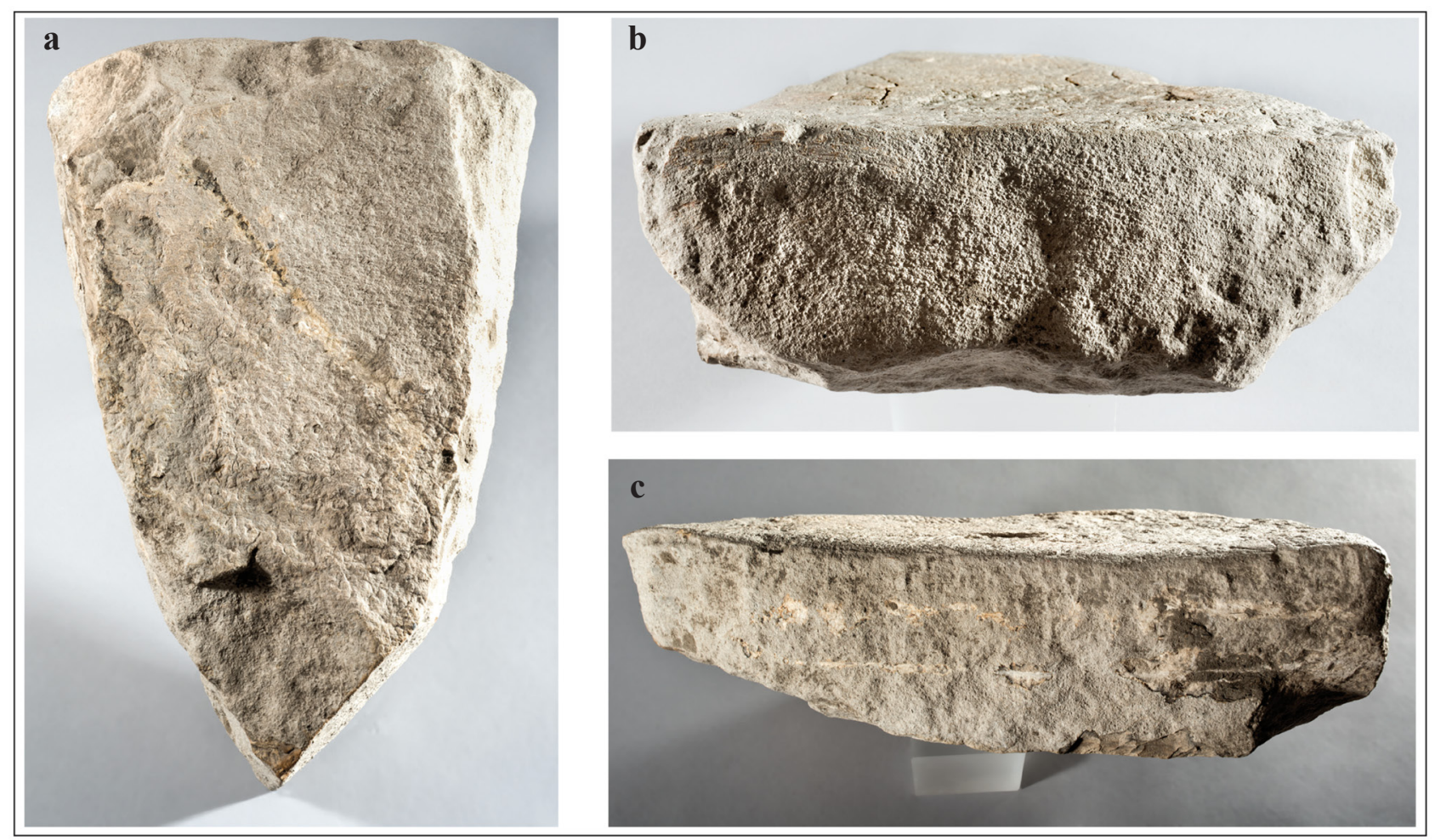

Fig. 5 - Gargas, stèle 1 de Beyssan, photographies de détail. a : la face dorsale est brute; $b$ : partie supérieure, traces du façonnage par raclage; $\mathrm{c}:$ la face principale montre une surface légèrement concave qui témoigne d'un travail de dressage préalablement à la réalisation du décor (cliché C. Durand, AMU-CNRS-CCJ).

Fig. 5 - Gargas, stela 1 from Beyssan, detail photographs. 1: back face is rough; 2: top part, traces of shaping by scraping; 3: front face shows a slightly concave surface which indicates pick-dressing work prior to the engraved ornamentation (photo C. Durand, AMUCNRS-CCJ).

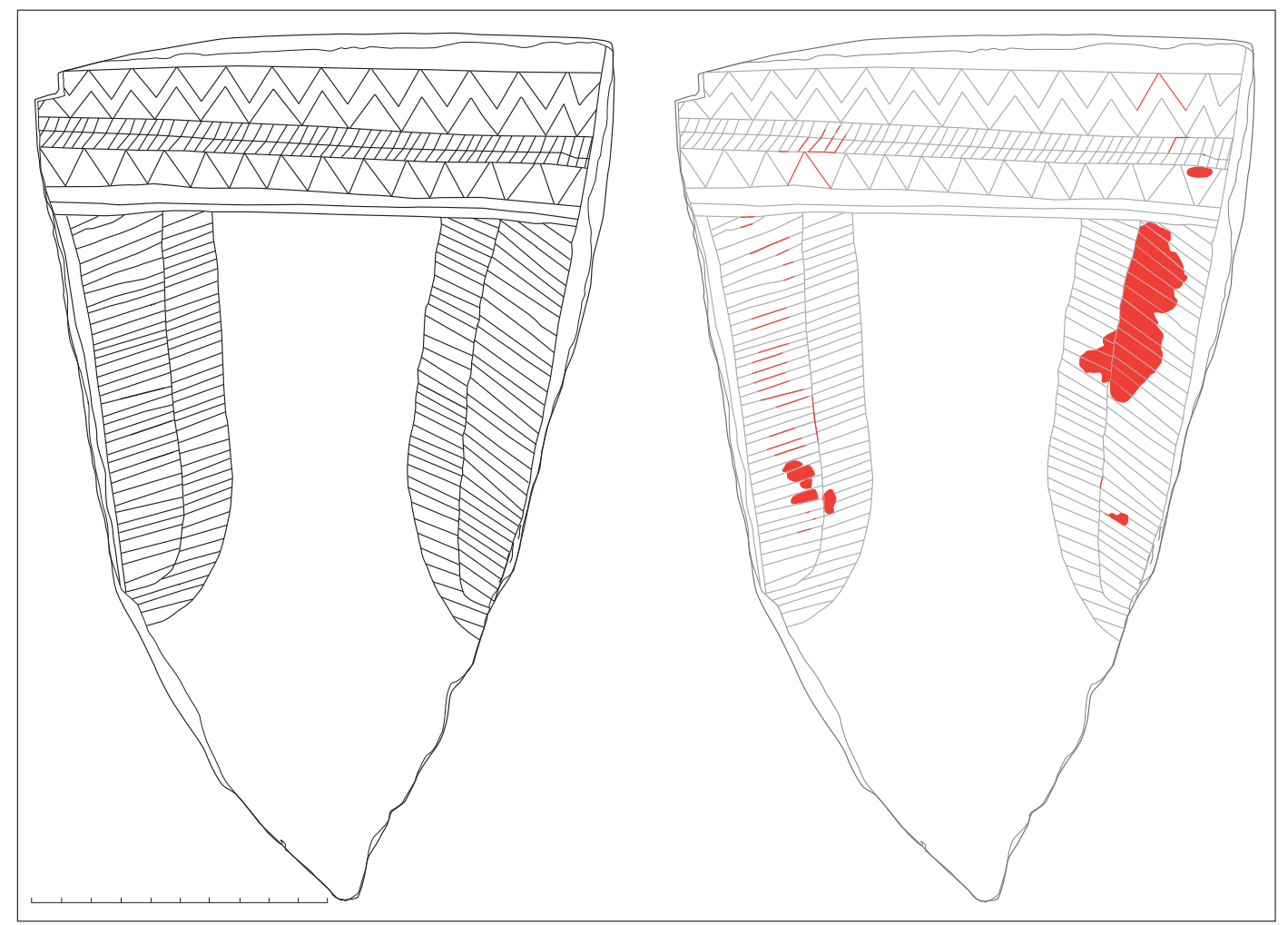

Fig. 6 -Gargas, stèle 1 de Beyssan, restitution du décor, localisation du pigment (dessin A. D’Anna).

Fig. 6 -Gargas, stela 1 from Beyssan, reconstruction of the ornamentation, location of pigment (drawing A. D'Anna). 
De bas en haut : 1) deux bandes horizontales lisses, plus hautes à gauche (5 et $6 \mathrm{~mm}$ ) qu'à droite (3 et $4 \mathrm{~mm})$; 2) une bande continue de chevrons simples horizontaux (A2), de 12 à $16 \mathrm{~mm}$ de haut; 3) deux bandes horizontales de hachures obliques irrégulières de droite à gauche (B2) de $5 \mathrm{~mm}$ de haut; 4) une bande de chevrons doubles horizontaux (A2 double) de $20 \mathrm{~mm}$ de haut.

Sur l'ensemble du décor gravé des traces de couleur rouge vif, brillantes, sont nettement visibles, tant dans les creux des gravures que sur les plats entre celles-ci, sur les parties latérales et sur la partie supérieure. La couleur est plus ou moins bien visible du fait de l'encroûtement qui la recouvre, mais il est probable qu'elle concerne l'ensemble des registres gravés. Afin de préciser l'étendue et la répartition du colorant, un premier examen de la surface de la stèle a été effectué, par Jacques Élie Brochier, sous DStrech. «Il s'agit d'un système de décorrélation des couleurs conçu à l'origine pour la télédétection ... adapté aux besoins des pariétalistes » (Le Quellec et al., 2009) pour l'amélioration des images d'art rupestre. « Il peut rendre visibles des pictogrammes presque imperceptibles à l'oeil nu. Il détecte des différences de teinte et les met en évidence ... »(Le Quellec et al., 2013). Cet examen avec des documents de qualité moyenne a montré que la partie centrale réservée semble exempte de colorant et que celui-ci est donc seulement présent sur les zones décorées, bandeaux supérieur et latéraux. Il montre aussi que le colorant incruste les hachures gravées et couvre les plats entre elles. L'examen des photos à haute résolution est en cours.

Un microprélèvement du pigment a été analysé par l'un de nous (L. B.-G.) au laboratoire MONARIS (UMR 8233, UPMC-CNRS) par spectroscopie Raman (voir ci-dessous). Les résultats obtenus indiquent qu'il s'agit de cinabre (sulfure de mercure, $\mathrm{HgS}$ ).

\section{La stèle 2}

La stèle 2 n'est pas totalement intègre car la base manque. La hauteur maximale conservée est de 19 à $20 \mathrm{~cm}$ et la hauteur initiale peut être évaluée à moins de $30 \mathrm{~cm}$; la largeur maximale en haut est de $17,5 \mathrm{~cm}$, la largeur de la face décorée en haut de $15,5 \mathrm{~cm}$, la largeur maximale en bas à la fracture est de $11 \mathrm{~cm}$; l'épaisseur maximale en haut est de $6,22 \mathrm{~cm}$, l'épaisseur maximale en bas de $6,46 \mathrm{~cm}$ (fig. 7, 8 et 9).

L'ensemble de la surface est très encroûté. Le matériau utilisé est différent de celui de la stèle 1 . Il est plus grossier, grumeleux, chargé en microfossiles et à cassure irrégulière. Il s'agit probablement d'un bloc de calcaire oligocène local ou du Crétacé inférieur des monts de Vaucluse. Là encore l'encroûtement ne permet pas une analyse précise. Les faces latérales et la face dorsale sont brutes mais montrent des traces d'aménagement par enlèvements d'éclats pour obtenir une forme subtriangulaire ou trapézoïdale. Les traces de la mise en forme ne sont pas visibles du fait de l'encroutement mais également de la nature grumeleuse du matériau. La mise en forme semble avoir principalement concerné la partie supérieure légèrement convexe et les bordures latérales à angles adoucis. La surface plate à localement convexe, ne semble pas avoir été dressée aussi soigneusement que pour la stèle 1. La face dorsale est subplane, naturelle, sans aménagement visible, avec un gros éclat ancien à la partie supérieure.

Le décor est gravé, rehaussé de couleur rouge vif. Il est plus simple et un peu moins soigné que celui de la stèle 1. Il n'y a pas de véritable relief. Bien que masqué par l'encroûtement sa lecture globale est possible et il peut être restitué (fig. 9). Il est organisé en trois zones autour d'une surface réservée verticale qui ne porte ni creux, ni relief sculptés, ni apparemment de couleur. Les attributs anatomiques, bloc sourcils-nez-yeux, ne sont donc pas représentés. La bande verticale a une largeur de $4,85 \mathrm{~cm}$ à $5,08 \mathrm{~cm}$ et une hauteur de $10 \mathrm{~cm}$ jusqu'à l'épaulement. Jusqu'à celui-ci les bordures sont verticales et ne montrent pas le resserrement observé sur la stèle 1. La partie inférieure est évasée (forme de visage de type 7, voir ci-dessus). Les bandes verticales latérales sont simples, larges de 2,0 à 3,4 cm, constituées de hachures obliques descendantes de l'intérieur vers l'extérieur (B2) de 0,6 à $0,7 \mathrm{~mm}$ de profondeur. Entre les hachures les plats sont assez larges et irréguliers $(0,50$ à $0,85 \mathrm{~cm})$.

La partie supérieure comporte deux bandes horizontales. Une bande étroite $(1,05-1,10 \mathrm{~cm})$ porte un décor difficilement lisible. Il s'agit soit de hachures de droite à gauche sur toute la largeur de la stèle, soit de hachures encadrant un cartouche de chevrons au-dessus de la zone réservée. Au-dessus, se trouve une frise de chevrons doubles (A3) de 2,60 à 2,85 $\mathrm{cm}$ de hauteur.

La structure globale de ce décor est conforme, la bande centrale est originale par sa forme et l'absence de figuration réaliste. Les motifs latéraux et supérieurs sont techniquement différents entre eux. Globalement, la stèle donne l'impression d'une moins grande finesse par rapport à la stèle 1. Là encore, les traces de colorant rouge sont nombreuses sur les bandes latérales, dans les creux et sur les plats, et sur le petit bandeau supérieur. Il ne semble pas y en avoir sur la bande centrale réservée. Une analyse par spectroscopie Raman a été réalisée sur un microprélèvement au laboratoire MONARIS (UMR 8233, UPMC et CNRS). Elle a permit, là également, d'identifier la présence de cinabre (sulfure de mercure, $\mathrm{HgS}$ ).

\section{Analyse des pigments}

Afin de déterminer la nature des matières colorantes observées dans les gravures et sur les surfaces du décor, il a été choisi de pratiquer des analyses non destructives par spectroscopie Raman sur quatre microprélèvements, deux par stèle, représentatifs des distributions de pigments.

\section{Méthode et conditions d'analyse}

Les analyses ont été réalisées avec un microspectromètre Raman HR800 Horiba Jobin Yvon. Elles ont été effectuées avec une excitation à $514 \mathrm{~nm}$ produite par un laser Argon ionisé. Afin d'enregistrer des spectres 


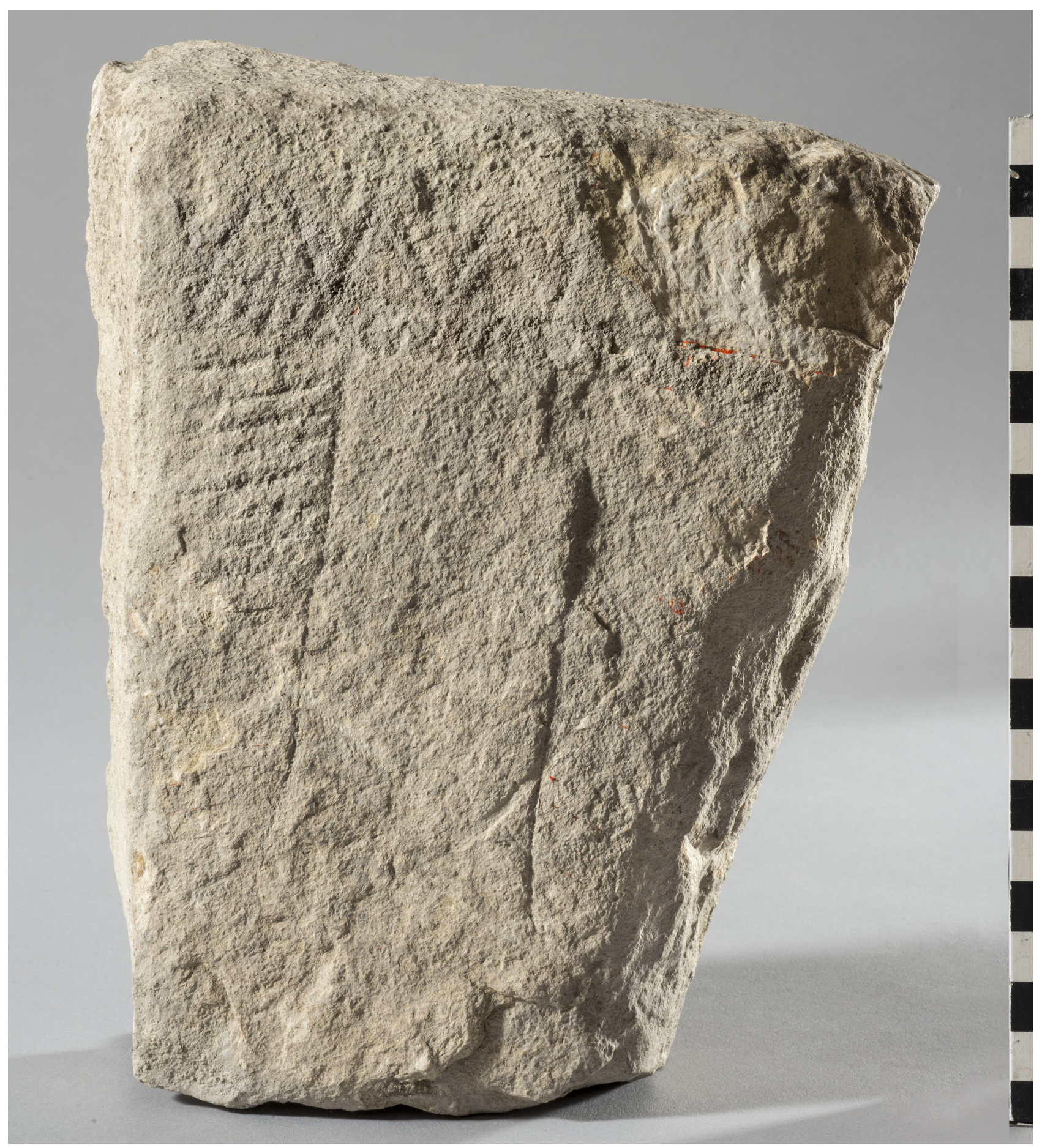

Fig. 7 - Gargas, stèle 2 de Beyssan (cliché C. Durand, AMU-CNRS-CCJ)

Fig. 7 - Gargas, stela 2 from Beyssan (photo C. Durand, AMU-CNRS-CCJ).

jusqu'à $10 \mathrm{~cm}^{-1}$ la réjection de l'émission Rayleigh est effectuée par trois filtres Notch BragGrate à fenêtre de coupure ultra-étroite (OptiGrate). Le signal est analysé par un réseau de 1800 traits $/ \mathrm{mm}$ donnant une résolution spectrale de l'ordre de $2 \mathrm{~cm}^{-1}$. La détection est réalisée à l'aide d'un détecteur CCD (Charge Coupled Device) refroidi par effet Peltier. Un microscope et ici un objectif $\times 100$ permettent de focaliser l'excitation laser et de récolter la diffusion Raman. Le volume de matière ainsi sondé est de l'ordre du micron cube.
Afin de préserver l'intégrité des échantillons, la puissance Laser est abaissée pour obtenir $100 \mu \mathrm{W}$ sur l'échantillon. Cette puissance particulièrement faible ne peut introduire d'altération ou de modification structurale des phases minérales étudiées.

\section{Échantillons et observations}

Sur chacune des deux stèles les deux prélèvements différents (identifiés $1 n^{\circ} 1,1 n^{\circ} 2,2 n^{\circ} 1$ et $2 n^{\circ} 2$ ) ont été 


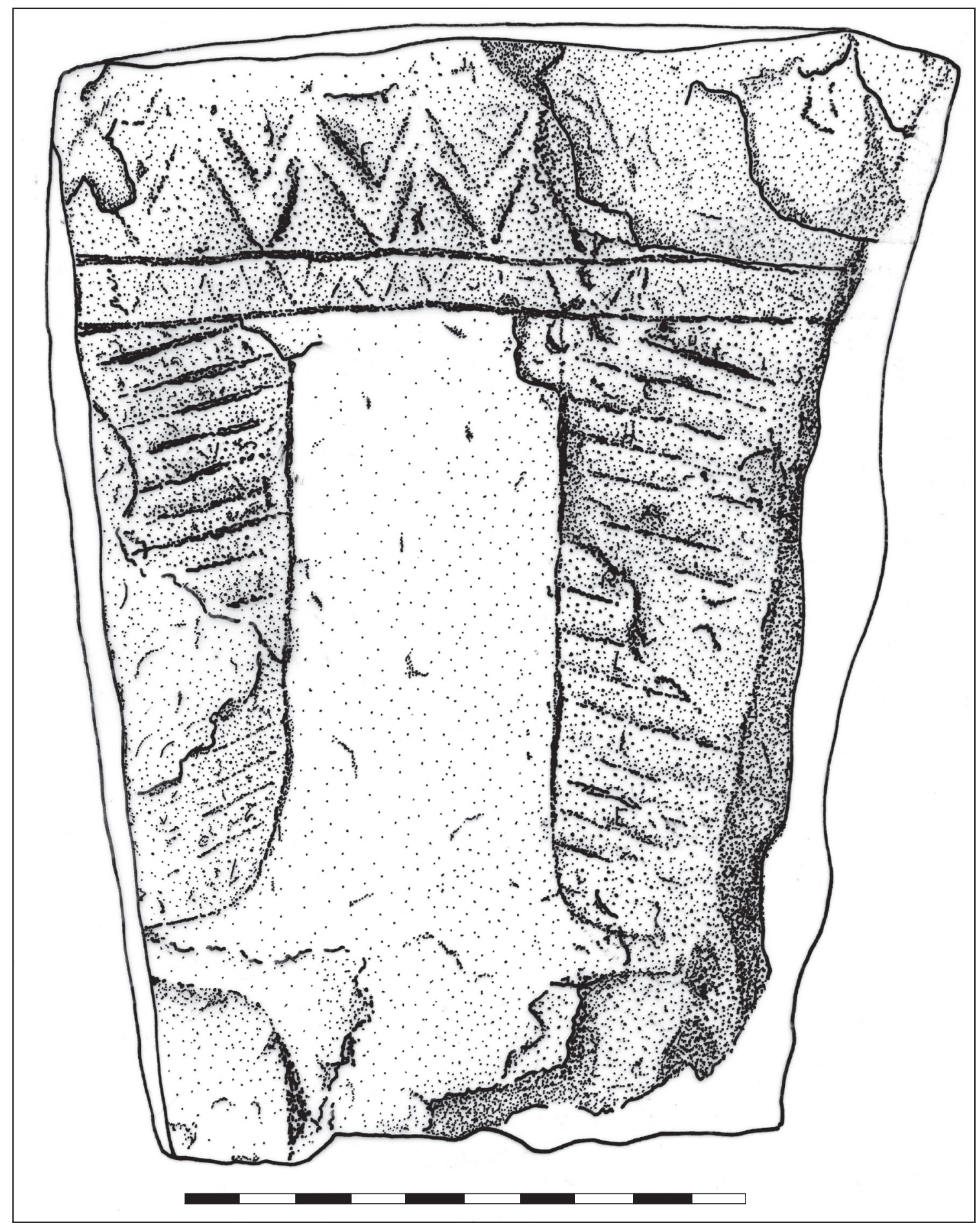

Fig. 8 - Gargas, stèle 2 de Beyssan (relevé et dessin A. D'Anna).

Fig. 8 - Gargas, stela 2 from Beyssan (drawing A. D'Anna)

effectués par C. Bosansky à l'aide de ruban adhésif sur les bandeaux supérieurs et sur le bandeau latéral de droite, zones de gravures où des pigments rouges étaient observables. Pour la stèle $n^{\circ} 1$ le prélèvement 2 ne présente pas de traces de pigments rouges. Pour les trois autres, les observations sous microscope optique montrent des microprélèvements riches en matière colorante de couleur vive (fig. 10a, b et c).
Résultats

Les analyses micro-Raman ont été effectuées directement sur les matières colorantes adhérentes au ruban adhésif.

Pour les deux stèles, les prélèvements présentant des matières colorantes rouges, des signatures Raman significatives ont été aisément obtenues. 


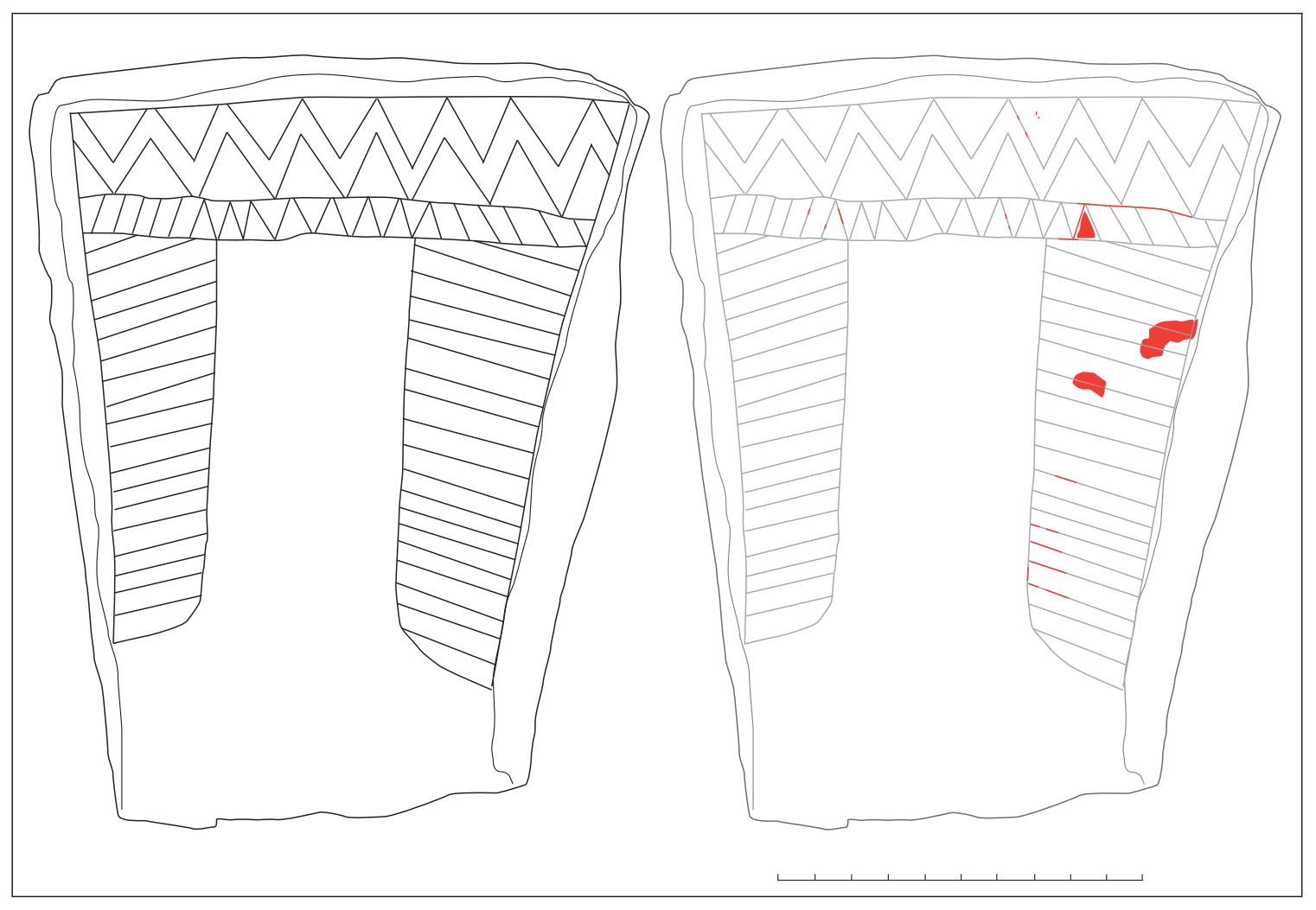

Fig. 9 - Gargas, stèle 2 de Beyssan, restitution du décor, localisation du pigment (dessin A. D’Anna).

Fig. 9 - Gargas, stela 2 from Beyssan, reconstruction of the ornamentation, location of pigment (drawing A. D'Anna).
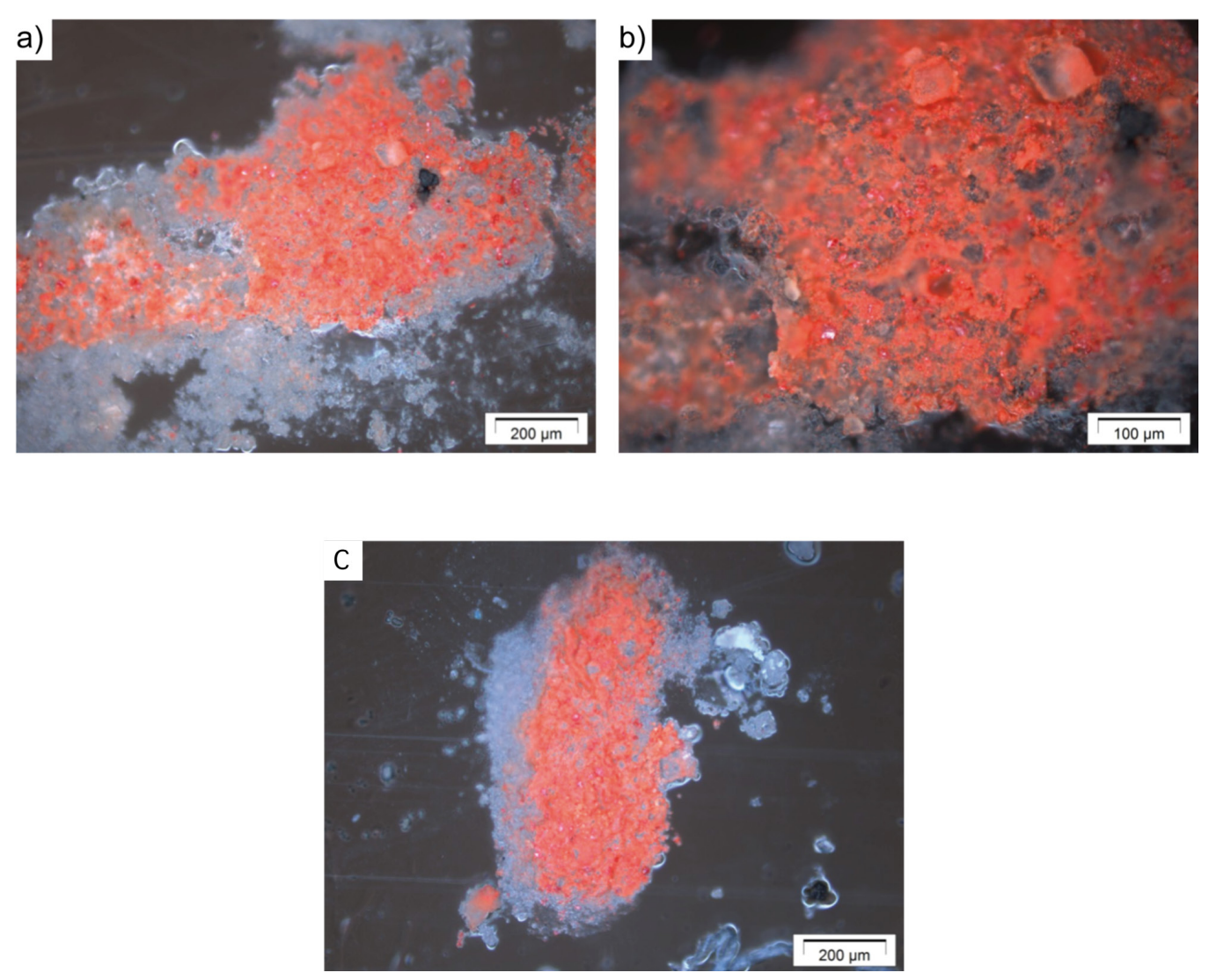

Fig. 10 - Gargas, stèles de Beyssan, vue sous microscope optique des prélèvements de pigment. a : stèle 1, $\times 10 ; \mathrm{b}:$ stèle $1, \times 20 ; \mathrm{c}:$ stèle $2, \times 10$. Fig. 10 - Gargas, stelae from Beyssan, optical microscope view of pigment sampling. a: stele 1, $\times 10 ;$ b: stele 1, $\times 20 ; c$ : stele $2, \times 10$. 
Les spectres sont dominés par une bande à $42 \mathrm{~cm}^{-1}$ (fig. 11). L'enregistrement de cette bande à basse fréquence $\left(<100 \mathrm{~cm}^{-1}\right)$ n'est possible qu'avec des caractéristiques instrumentales particulières du spectromètre Raman, comme celles utilisées pour cette étude. Une seconde bande est aisément observable à $254 \mathrm{~cm}^{-1}$, les autres, d'intensités moindres, ne sont pas clairement visibles avec l'échelle utilisée. Ces spectres correspondent à la signature structurale du cinabre, sulfure de mercure, HgS (Dawson, 1972; Gotoshia et Gotoshia, 2008). Pour illustrer cette identification, la signature structurale d'un échantillon de référence de cinabre issu des mines d'Almadén (Espagne) est également présentée.

C'est donc du cinabre qui a été utilisé pour produire la coloration rouge présente dans les prélèvements étudiés.

\section{COMPARAISONS, CONSÉQUENCES ET APPORTS}

\section{Inventaire et géographie}

À la suite des dernières découvertes d'Aix-en-Provence (Hassler, 2014), Miouvin (Sauzade et Cerclier, 2014) et Oraison (inédit, renseignement oral de X. Margarit), les stèles de Gargas sont les $52^{\mathrm{e}}$ et $53^{\mathrm{e}}$ stèles néolithiques provençales. Ces dernières proviennent de dix-huit sites. Le total de l'inventaire ne donne qu'un nombre minimum puisque à La Bastidonne, plusieurs petits fragments portant des chevrons gravés ont été recueillis, mais ne sont

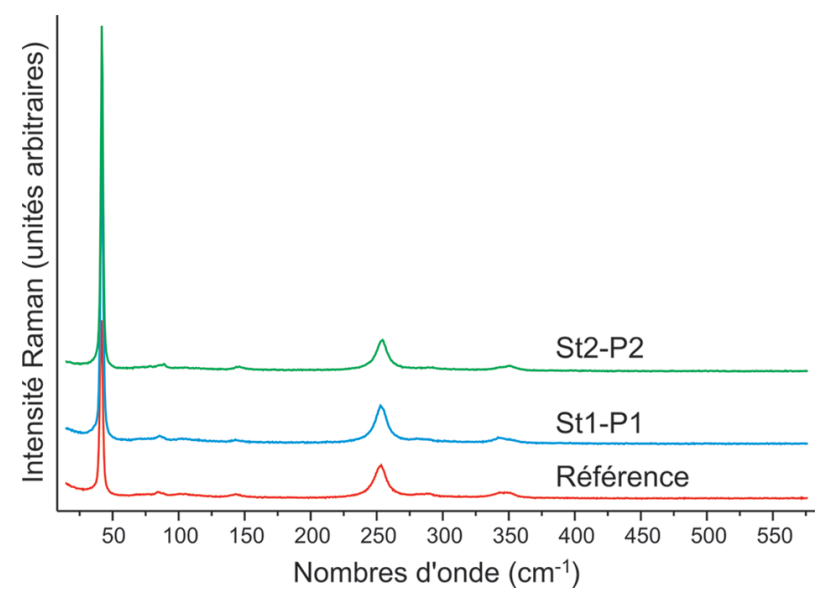

Fig. 11 - Gargas, stèles de Beyssan, spectres Raman représentatifs des signatures obtenues sur les prélèvements de pigment des deux stèles ( $\mathrm{St} 1$ et $\mathrm{St} 2$ et respectivement pour les prélèvements 1 et 2). Ils sont comparés avec un spectre obtenu dans les mêmes conditions sur un échantillon de référence de Cinabre d'Almadén (Espagne).

Fig. 11 - Gargas, Stelae from Beyssan, Raman spectra showing representative signatures from pigment samples from both stelae (St 1 and St 2 and respectively for samples 1 and 2). For comparison, a spectrum acquired under the same conditions on a reference sample of Cinnabar from Almadén (Spain) is shown. pas pris en compte du fait de leurs petites dimensions (D’Anna et al., 2004). Il est également probable que sur ce site d'autres ramassages aient été effectués. Sans aucun doute la Bastidonne à Trets devait donc compter plus de stèles que les douze fragments conservés au musée des Antiquités nationales et du fragment signalé par M. Escalon de Fonton. Par ailleurs, les aventures des stèles de Cavaillon (D'Anna et al., 1997b), du Beaucet et de Miouvin (Sauzade et Cerclier, 2014) laissent supposer, et craindre, que quelques autres beaux exemplaires peuvent exister ici où là sans avoir été signalés ou vus par des archéologues ...

La localisation de la découverte (fig. 1), loin de la Durance, au nord du Luberon, sur la marge nord de la plaine du Calavon, sans être surprenante, est originale. Elle vient s'insérer dans la partie nord de l'aire de répartition des stèles provençales, entre les stèles de Goult (Gagnière et Granier, 1977-1978; Sauzade et al., 2003) à 12,5 km au sud-ouest, les stèles du Beaucet (Sauzade, 1987) à un peu plus de $20 \mathrm{~km}$ au nord-ouest, et les stèles de Villeneuve (Corseaux et al., 1984) et Oraison à un peu plus de $40 \mathrm{~km}$ à l'est. Cela confirme que plusieurs stèles sont liées à la vallée du Calavon, comme d'autres le sont à celles de l'Arc et de la Durance. Cela justifierait de rebaptiser l'ensemble des stèles provençales des groupes $\mathrm{B}$ et $\mathrm{C}$ (voir ci-dessous) stèles du groupe en pierre Durance-Arc-Calavon. La découverte des stèles de Gargas n'étend pas la région de répartition mais contribue à la densifier et confirme que les stèles à chevrons constituent apparemment un phénomène localisé dans l'espace.

\section{Iconographie, stèles à chevrons et stèles peintes}

À la suite des travaux de Sylvain Gagnière, il était de tradition de classer les stèles provençales en deux groupes (Gagnière et Granier, 1963 et 1976).

Le groupe A, constitué de stèles en « forme de borne, à base plane permettant de les poser sur le sol. Le dessin est déterminé par des lignes courbes, avec sourcils arrondis, il n'y a pas de décoration, mais on observe la présence de nombreuses cupules. Les motifs, figurations humaines ou cupules, occupent les deux faces et les yeux sont toujours traités en creux. Enfin, la matière employée pour ces sculptures est la mollasse calcaire ... ». Ce groupe comporte trois monuments : Avignon, rocher des Doms, Avignon, la Balance et L'Isle-sur-la-Sorgue, la Grande Bastide, qui constituent chacun un type; sa connaissance n'a pas progressé depuis 1976, date de la dernière découverte le concernant. Ce groupe a été également dénommé groupe venaissin ou stèles venaissines (Arnal, 1976a; D'Anna, 1977) ou encore stèles à cupules (Sauzade, 1983) sans en changer le contenu. Il semble actuellement que ces monuments puissent représenter une extension orientale du groupe languedocien et être datés de la fin du Néolithique (D’Anna et al., 1997) comme cela a toujours été proposé. En effet, on trouve quelques éléments de comparaison avec les stèles languedociennes. Leur forme générale en borne et leurs dimensions modestes, 
entre 26 et $46 \mathrm{~cm}$ pour les deux exemplaires entiers, sont comparables à celles de Saint-Théodorit, les Roumanis, et Euzet, le Colombier, dans le Gard. La forme du visage et du bloc nez-sourcils sont comparables à celle des stèles gardoises de Bon Diablet à Saint-Quentin-La-Poterie et de la Maison-Ferrand à Blauzac (Jallot, 1987; Gutherz et al., 1998) ou de la stèle héraultaise du Truc de Marti à Viols-le-Fort (Rouquette, 1998). De plus, à la fin du Néolithique, période pendant laquelle elles sont sculptées, leur région de découverte, dans la basse vallée de la Durance, est bien en domaine Ferrières puis Fontbouisse (Gutherz et Jallot, 1999; Jallot, 2011). Les stèles de ce groupe venaissin ne seront donc pas prises en compte dans les comparaisons et discussions.

Dans la définition initiale (Gagnière et Granier, 1963) le groupe B était « caractérisé par des stèles plus ou moins plates, avec large sommet horizontal et extrémité inférieure destinée à être fichée en terre. Le dessin est déterminé par des lignes droites, avec sourcils rectilignes. Il y a presque toujours une décoration géométrique (en général des chevrons) qui (...) encadre le visage, la cupulation est inexistante... Les yeux lorsqu'ils sont figurés sont traités en léger relief ou indiqués en creux. Le visage, rectangulaire, est presque toujours en champlevé. La sculpture n'occupe qu'une seule face de l'objet et la matière employée est le calcaire dur à grain fin ». Ce groupe a été par ailleurs baptisé « groupe de basse Durance » (Arnal, 1976a) ou « des stèles à décors de chevrons ou encore durancien » (D'Anna, 1977). Initialement il était composé de trois types : type 1 « caractérisé par la présence des yeux et des avant-bras »; type 2 " semblable au précédent et ne s'en distingue que par l'absence des yeux »; type 3 « absence des yeux et des avant-bras ». Là encore la définition reste totalement d'actualité, mais depuis 1963, les nouvelles découvertes de Lauris-Puyvert ont permis de la compléter avec la présence de sommets concaves (Gagnière et Granier, 1967 et 1976), de sourcils et de limites du visage en courbes (Villeneuve, le Beaucet) et de quelques exemplaires en molasse (Cavaillon; Goult, Ponty-sud). La distinction formelle entre les trois types, compte tenu du nombre accru d'exemplaires n'est probablement plus strictement possible. D'un point de vue de détail, il a été suggéré d'abandonner la dénomination « avant-bras », comme le proposait déjà Octobon : « il n'y a pas de bras, car on ne peut interpréter ainsi ni les finales frangées des dessins qui les couvrent, ni les deux tenons qui limitent vers la partie inférieure la surface polie ... la tête étant figurée en creux par la partie rectangulaire qui porte les yeux et le nez, ... et le cou était indiqué par les deux tenons qui séparent la tête du reste du corps » (Octobon, 1931, p. 468). Dans cette perspective ces stèles ne représentent qu'un visage. On pouvait en distinguer six formes (D'Anna, 1977, p. 214; D'Anna et al., 2004) : 1) visage complet avec yeux, nez, cou en creux et épaules en relief; 2) visage complet avec cou mais ni les yeux ni le nez; 3) visage sans yeux, nez, cou et bas fermé; 4) visage non limité et matérialisé seulement par le bloc nezsourcils; 5) visage schématique sans détail anatomique à bordures anguleuses; 6) visage schématique à bordures convergentes.
L'organisation des éléments de base du décor, gravé ou sculpté en champlevé, permet de reconnaître différents motifs :

- Le chevron simple constitue l'un des deux motifs de base (A1).

Multiplié, il est décliné en lignes (A2) et bandes (A4) puis surfaces (A3 et A5). La ligne est constituée de chevrons juxtaposés. L'association de lignes constitue une surface continue (A3). S'ils sont emboîtés, les chevrons constituent des bandes dont la juxtaposition donne également une surface continue (A5). Les bandes peuvent être margées (A6) et constituer à leur tour, par juxtaposition, des surfaces de bandes margées (A7). Lignes et bandes peuvent être verticales ou horizontales.

- Les hachures constituent le second motif de base (B1).

Elles sont généralement margées, ce qui constitue des bandes de hachures (B2). Les bandes juxtaposées donnent des surfaces de hachures margées ou des surfaces de bandes de hachures (B3). La juxtaposition de bandes de hachures d'orientations différentes constitue des motifs en « arêtes de poisson » (B4) ou des surfaces « d'arêtes de poisson » (B5). Là encore les bandes peuvent être horizontales ou verticales.

Dans tous les cas, les oppositions d'orientations déterminent des losanges ou des triangles réservés. La combinaison des motifs élémentaires en jouant sur leur juxtaposition et sur les orientations permet une variabilité de l'ensemble des décors qui contribue à rendre illusoire un classement typologique strict. Et ce d'autant plus qu'au moins deux exemplaires, Sénas 1 et Aix-en-Provence, ne portent pas de décor de chevrons mais seulement la forme du visage.

Depuis 1976, un troisième groupe, que l'on peut dénommer $\mathrm{C}$ ou stèles lisses à décor peint ou encore stèles de type Château Blanc, a été reconnu, avec les sept stèles de la première phase de Château Blanc à Ventabren (Hasler, 1998; Hasler et al., 1998 et 2002), et les deux stèles trouvées sous le dolmen l'Ubac à Goult, (Sauzade et al., 2003; Sauzade, 2012). Elles sont soigneusement mises en forme, ne portent pas de décor gravé mais montrent des traces de pigment rouge qui à Château Blanc a été identifié comme de la bauxite (Walter et al., 1997). Les stèles découvertes en réemploi dans le dolmen du Pouget dans l'Hérault (Arnal et al., 1986) à plus de $150 \mathrm{~km}$ à l'est et conservées au musée de Lodève, appartiennent indéniablement à la même famille.

Les deux stèles de Gargas peuvent être rattachées au groupe B. Elles en confirment à la fois la diversité individuelle et l'unité globale; si elles sont totalement conformes à la typologie du groupe, elles présentent cependant quelques originalités qui permettent encore une fois de préciser la définition générale du groupe, mais conduisent également à y créer un nouveau type ou sous type.

\section{Forme et dimensions}

La forme triangulaire de la stèle 1 de Beyssan s'intègre parfaitement à la variabilité des formes des stèles à chevrons. Elle est proche de celles de Lauris-Puyvert 1 et 2 , 
Mont-Sauvy, le Beaucet 1, Trets 2, 3 et 4. On notera que c'est ici un triangle bien marqué comme pour Trets 5 .

La forme de la stèle 2, subrectangulaire pourrait paraître plus originale, mais cela vient du fait que la base est cassée, sa forme est plus proche de celles des stèles de Sénas.

En revanche le sommet légèrement convexe est particulier puisqu'il ne se rencontre que sur deux exemplaires de Château Blanc et sur une des deux stèles de l'Ubac à Goult, .

Les hauteurs des deux stèles de Gargas sont un peu au-dessous de la moyenne, moins de $30 \mathrm{~cm}$ dans les deux cas ; Lauris-Puyvert 1 (32 cm), Lauris-Puyvert $2(35 \mathrm{~cm})$, Cavaillon $(33 \mathrm{~cm})$, Orgon Mont-Sauvy $(37 \mathrm{~cm})$ et Fond de Malte $(44 \mathrm{~cm})$, sont un peu plus grandes; Orgon 7 $(28 \mathrm{~cm})$ est un peu plus petite et Orgon 2 devait avoir la même hauteur ou à peine plus $(30 \mathrm{~cm})$. Avec Gargas 1 il n'y a que sept stèles entières qui donnent une moyenne de $34 \mathrm{~cm}$ de haut, entre 28 (Orgon 7) et $44 \mathrm{~cm}$ (Font de Malte). Cependant cela reste trompeur, si l'on tente une intégration des stèles brisées de Sénas (Sénas 4 environ $40 \mathrm{~cm}$, Sénas 5 environ $40 \mathrm{~cm}$, Sénas 3 environ $45 \mathrm{~cm}$ et Sénas 7 environ $45 \mathrm{~cm}$ ), la moyenne des hauteurs est augmentée à $37 \mathrm{~cm}$ ! Et encore plus avec la stèle du Beaucet 2 , de plus de $50 \mathrm{~cm}$ de haut, découverte en 1993 (Sauzade et Cerclier, 2014). Nous avons pu examiner en détail cette stèle en 2005 grâce à l'amabilité de M. Castan, que nous remercions de cette initiative, et c'est sur ces observations que nous nous fondons ici. Bien qu'il soit difficile d'en juger, on doit considérer que la stèle de Villeneuve (plus de $45 \mathrm{~cm}$ de hauteur) et celles de Trets étaient également plus grandes (Trets 1, 3 et 5 par ex., plus de $45 \mathrm{~cm}$ ). Enfin, les stèles du type de Château Blanc et l'Ubac étaient nettement plus grandes, environ $60 \mathrm{~cm}$ à l'Ubac, et entre 55 et $80 \mathrm{~cm}$ à Château Blanc, mais l'une d'elles, incomplète, pouvait avoir plus de $90 \mathrm{~cm}$ (TIII 3267).

Les matériaux utilisés, calcaire oligocène pour la stèle 1 et calcaire oligocène ou crétacé pour la stèle 2 , sont conformes à ce qui était déjà connu, c'est à dire exclusivement des calcaires tendres de préférence aux autres calcaires plus durs abondants dans la région (D'Anna et al., 2004). À Gargas, leur origine, comme cela a été également remarqué dans la majorité des cas, n'est pas très éloignée du site de découverte mais n'est pas strictement locale. La différence de nature des matériaux utilisés à Gargas confirme des ramassages opportunistes plutôt que des extractions en carrière.

\section{Le décor : le visage et les chevrons}

La structure globale du décor est conforme à ce qui était connu, mais dans le détail il y a deux aspects qui n'existaient pas dans la typologie établie (D'Anna, 1977; D'Anna, et al., 2004).

La forme du visage des deux stèles de Gargas est nouvelle, sa partie supérieure est horizontale comme sur toutes les stèles, mais elle ne fait apparaître ni le nez, ni les yeux, ce qui n'était le cas que pour les stèles de Trets. Sur la stèle 1 la bordure du visage se resserre vers le bas puis la courbe s'inverse et s'évase vers l'extérieur, ce qui est également le cas pour la stèle 2 . Cela fait référence à la stèle du Beaucet 2 et probablement également à Beaucet 1 . L'association de ces deux détails, partie supérieure du visage horizontale sans nez et partie latérale courbe est originale. Elle doit être rapprochée de certaines stèles de Trets sur lesquelles on note également d'une part l'absence de représentation des motifs anatomiques yeux-nez et d'autre part des limites des zones réservées localement courbes.

Dans le décor de chevrons, on remarque qu'il y a une différence de structure entre les bandeaux latéraux et supérieurs. La rupture est nettement matérialisée au niveau du haut du visage par le passage de registres verticaux à des registres horizontaux à la partie supérieure.

Jusqu'ici une telle différence de structure, avec changement d'orientation du décor et limite marquée, dont le meilleur exemple est Font de Malte à Orgon, était faiblement minoritaire; en revanche à Lauris-Puyvert 2, Mont Sauvy et la Bastidonne 1, il y a un changement d'orientation mais pas de rupture marquée, alors qu'à Lurs il a une rupture marquée par une petite ligne réservée, mais le motif est en quasi continuité sans changement d'orientation. Pour les autres, il y a continuité totale entre les bandeaux latéraux et supérieurs : Lauris-Puyvert 1, Cavaillon, Le Beaucet 1, Sénas 2 et 7.

Enfin pour ce qui concerne le mode de réalisation du décor, on notera que celui-ci était jusqu'ici considéré comme obtenu par champlevé (Gagnière et Granier, 1963, 1976; D’Anna et al., 2004). À Gargas ce n'est pas le cas, il y a seulement gravure sur une surface soigneusement dressée (stèle 1) ou simplement régularisée (stèle 2). La surface « du visage » n'est pas en creux. Ce mode de réalisation se retrouve sur les stèles 5 et 6 de la Bastidonne. L'épais encroûtement ne permet pas de reconnaître un traçage préalable du décor comme cela a été observé sur les stèles de Sénas (la Puagère 3, 4, 7), Orgon (Font-deMalte), Le Beaucet 1 (D'Anna et al., 2004). À propos de la réalisation, on peut également remarquer que les deux stèles de Gargas semblent montrer des « mains différentes », l'une très précise (stèle 1), l'autre un peu moins habile (stèle 2).

Dans tout le Midi la présence du décor de chevrons est limitée à la Provence, en dehors des ceintures de certaines des statues-menhirs du Rouergue (Hermet, 1898; Octobon, 1931; Arnal, 1976a et b; D'Anna, 1977; Philippon, 2002). La signification de ces chevrons a été l'origine de différentes hypothèses d'interprétation symbolique et décorative (Gérin-Ricard, 1910; Gérin-Ricard et Arnaud d'Agnel, 1907) ou réaliste : coiffe ou plus probablement cheveux (Escalon de Fonton, 1962; Gagnière et Granier, 1963 et 1976; Arnal 1976a ; D’Anna, 1977) et que l'on peut résumer par « chevelure, résille, coiffure d'apparat, voire cape $»$ (Guilaine, 2003). Aucune de ces hypothèses ne peut trouver de confirmation définitive.

Ce type de décor de chevrons, hachures et zigzags, existe également sur des stèles ou dans l'art mégalithique de différentes régions, plus ou moins éloignées ou distantes de la Provence. Par exemple, en Catalogne, sur le remarquable ensemble de grandes stèles de Reguers de 
Sero récemment découvert en réemploi dans un monument utilisé par des porteurs de vases campaniformes (Lopez et al., 2009). Une seule est entière, mais les fragments conservés sont suffisamment importants pour montrer que les chevrons sont ici répartis en grandes surfaces occupant la quasi totalité d'une des deux faces et parfois les chants. Dans une autre direction, en Allemagne, des chevrons sont gravés sur la dalle de chevet et sur la dalle d'entrée du monument de Züschen I en Hesse, prés de Kassel (Anati et Varela Gomes, 2013). Également en Allemagne, des chevrons sont figurés sur la paroi des monuments de Göhlitzsch et Halle-Dölauer (Müller, 1997) et sur la stèle d'Ellenberg (Häusler, 1966). Enfin, sur les stèles de Sion et Aoste (Gallay, 1995; Mezzena, 1998; Corboud, 2009), les motifs de losanges et triangles finement gravés sont dans le même esprit. Tous ces exemples sont éloignés dans le temps (voir ci-dessous) et dans l'espace des stèles provençales, mais dans tous les cas ces motifs en surface continue ou en bande sont interprétés comme des tissus ou des vêtements.

\section{De la couleur}

La détermination de l'utilisation du cinabre sur les stèles de Gargas conduit à s'interroger à plusieurs niveaux.

La présence de pigment rouge a été très tôt identifiée sur les stèles de Trets, la Bastidonne, sur lesquelles les détails du visage étaient initialement considérés comme peints (Cotte, 1905; Gérin-Ricard, 1910). Pour les stèles de Sénas, la Puagère, le commandant Octobon avait également précisé : « ces dalles paraissent avoir été peintes en ocre et en rouge » (Octobon 1931, p. 368). Bien que sur le fond blanc les surfaces de couleur rouge soient particulièrement évidentes à l'œil nu, aucun examen détaillé n'avait été entrepris. Ces traces de couleur sont dans tous les cas très localisées et mal conservées. Sur la stèle du Beaucet 1 , les traces de colorant sont nombreuses, elles ont d'abord été considérées par observation visuelle comme de l'ocre (Sauzade, 1987) puis déterminées comme du cinabre (Sauzade et Cerclier, 2014), c'est probablement également le cas pour la stèle 2 .

La première étude détaillée sur cette question des colorants, avait permis de déterminer l'utilisation d'une bauxite sur quatre stèles de Ventabren, Château Blanc (TI, 3248, 3249, 3250 et TIII 3198) et de cinabre sur les stèles 1 et 4 (soit 4 et 2 de la numérotation D'Anna) de Trets, la Bastidonne (Walter et al., 1997).

À l'occasion de la préparation de la réédition du catalogue des stèles du musée Calvet d'Avignon, il avait été possible de réaliser plusieurs nouveaux prélèvements afin de déterminer la nature des pigments visibles sur quatre stèles de Sénas, la Puagère : stèles 1, 3, 4 et 5. Sur ces stèles, les zones rouges ne montrent pas de localisation particulière et il ne semble pas que la couleur pouvait servir à préciser la représentation du visage. La nature du pigment a été identifiée par P. Walter, après observation au microscope optique et analyse au microscope électronique à balayage couplé à un détecteur de rayons X. Dans tous les cas il s'agissait d'ocre (D'Anna et al., 2004).
Actuellement, la présence de colorants a donc été reconnue sur vingt-quatre stèles et ils ont été déterminés pour treize d'entre elles. Ils ont été nommés ocre dans quatre cas (la Puagère 1, 3, 4 et 5) et bauxite dans quatre cas (Château Blanc) et déterminés comme du cinabre dans six cas (la Bastidonne 2 et 4, le Beaucet 1 et 2, Gargas 1 et 2). On peut constater ainsi que, dans l'état des déterminations, chaque site ne livre qu'une nature de pigment. Si l'on propose, par hypothèse, d'étendre ce constat aux stèles dont le pigment n'a pas été déterminé, le cinabre pourrait être dominant principalement du fait de sa présence à la Bastidonne.

Les pigments utilisés sur les stèles provençales sont donc de natures différentes (fig. 12). La présence de bauxite et d'ocre n'est pas surprenante dans la région. Ce sont ces pigments qui ont été également déterminés dans l'art rupestre postglaciaire de la Drôme et du Var (Hameau et al., 1995 et 2001).

Il n'en est pas de même du cinabre qui est relativement rare dans la nature et d'utilisation peu commune pendant la Préhistoire dans le Sud-Est de la France. Les questions du sens de son usage et de son origine pourraient être les premiers niveaux d'une discussion originale considérant que dans la région de Gargas les sources principales des pigments rouges sont constituées par des oxydes de fer présents dans diverses altérites. Le cinabre est un sulfure de mercure, et un des plus importants minéraux qui permettent d'obtenir ce métal. Il est également utilisé comme pigment : le vermillon (Hochleitner, 1984; Duda et Rej1, 1986; Bishop et Hamilton, 2005). Ses sources peuvent être diverses, dans des veines ou des incrustations dans des terrains calcaires ou argileux, mais principalement à proximité de phénomènes volcaniques. Les mines les plus connues sont celles d'Almaden en Espagne près de Ciudad Real à $200 \mathrm{~km}$ au sud de Madrid dans la Sierra Morena, du mont Amiata en Toscane, proche de Grosseto et Sienne, et d'Idrija, en Slovénie. Si l'on cherche au plus près du Vaucluse et de la basse Provence de petits gisements sont signalés près de Pessens (Aveyron), de Clermont-l'Hérault et de Tuchan (Aude), près de Chalanches et d'Allemont en Isère (Muséum national d'histoire naturelle, 2015, site internet) et au cap Garonne dans le Var (Syndicat intercommunal de la mine de Cap Garonne, 2015, site internet) qui serait la source possible la plus proche de Gargas, de Trets et du Beaucet. Cependant des études et vérifications du potentiel de ces différentes localités pour fournir du cinabre exploitable par les Néolithiques restent à entreprendre. On doit également signaler ici la présence de cinabre en Sardaigne : dans différentes mines et grottes de l'Iglesiente, par ex. la grotte San Giovanni à Domusnovas à l'est d'Iglesias ou encore la grotte de Corona 'e Sa Craba à l'est immédiat de Carbonia, dans les mines du Monte Zippiri, près de Villasor et de Serramanna, en bordure occidentale du Campidano, à environ $35 \mathrm{~km}$ au nord-ouest de Cagliari et à environ $40 \mathrm{~km}$ au sud du Monte Arci, et sur la bordure orientale des Monti Linas (Baldoni et al., 2013). Cette origine est évidemment sérieusement à prendre en compte du fait de la présence d'obsidienne sarde sur le site (voir ci-des- 


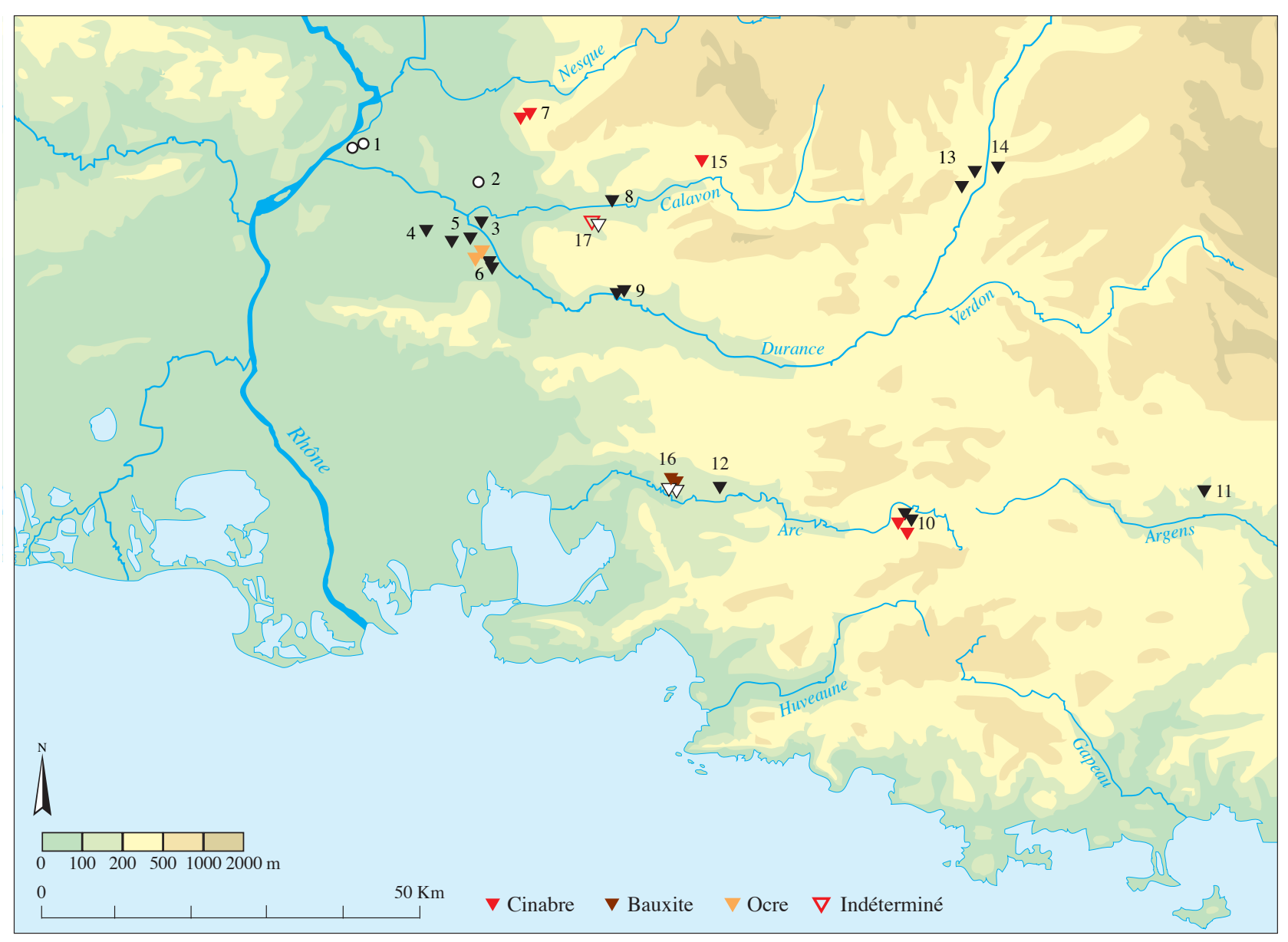

Fig. 12 - Nature des pigments identifiés sur les stèles provençales, carte de répartition (sites : voir fig. 1).

Fig. 12 - Nature of the pigments identified on the Provence stelae, distribution map (sites: see fig. 1).

sous). La diffusion de ce verre volcanique est considérée depuis longtemps comme associée à d'autres matériaux (Courtin, 1973; Vaquer, 2007).

\section{Contextes et chronologie}

\section{Contexte des stèles de Beyssan}

Les stèles de Gargas ont certes été recueillies en surface à la suite d'un labour, mais les ramassages de mobilier associé indiquent qu'il y avait là un contexte relativement précis. Probablement très mal conservé du fait de la localisation du site et des labours successifs, ce dont témoigne l'abondance du mobilier lithique en surface. L'ensemble constitue une série particulièrement spectaculaire comme il en existe plusieurs en Vaucluse (Courtin, 1974; Marchesi, 1990; Léa et al., 2004). Il provient de plusieurs locus autour de la campagne Beyssan. Différentes zones de ramassage ont été distinguées : Apt, Beyssan Cerisiers, au sud-est de la maison, Gargas, Beyssan sud, immédiatement devant la maison, Apt, Beyssan Julien, au nord-est et Gargas, Beyssan-NO, d'où proviennent les deux stèles (fig. 2). La discontinuité constatée témoigne probablement de la variation de densité des vestiges, mais elle est en grande partie artificielle et doit résulter de la conservation inégale des différents secteurs et des conditions de ramassage. Quoi qu'il en soit ces vestiges proviennent d'un même très vaste établissement (les points les plus éloignés le sont de près d'un kilomètre) évoluant dans une période relativement courte.

Un premier examen de l'ensemble montre une grande homogénéité qui autorise, en attendant une étude plus précise et détaillée, quelques remarques globales.

La céramique est quasi absente en dehors de quelques tessons portant des petits mamelons circulaires ou allongés.

L'industrie en silex taillé en revanche est particulièrement abondante. Le matériau utilisé est très majoritairement du silex «blond » barrémo-bédoulien du Crétacé de la région de Murs à environ $12 \mathrm{~km}$ au nord-ouest, matière première dont on connaît l'utilisation préférentielle pendant le Néolithique moyen (Courtin, 1974; Binder, 1984, 1991 et 1998; Léa et al., 2004). De nombreux fragments sont patinés partiellement ou entièrement (cacholong) et, ou, brulés. Il y a quelques autres natures de silex quasiment anecdotiques : du brun et, ou, zoné de type Forcalquier et un matériau rouge-lie de vin à taches blanches, quartzite crétacé ou éocène de type Pié Bousquet. Ce matériau original, souvent dit avec gourmandise « mortadelle du Calavon », est connu à quelques exemplaires sur 
tous les sites néolithiques du bassin d'Apt. Il provient des faciès dit des ocres d'Apt liés à des sols rouges silicifés.

À Beyssan-NO, on notera plus particulièrement la présence de deux fragments de nucléus quadrangulaires plats chauffés et d'un fragment de nucléus conique non chauffé, des lames et lamelles et fragments de lames et lamelles de différents modules mais régulières et de petites dimensions (largeur $1,5 \mathrm{~cm}$ à $2 \mathrm{~cm}$ et rarement plus) et provenant de nucléus chauffés ou non. Il y a également quatre armatures tranchantes, deux triangulaires à retouche plate envahissante et deux trapézoïdales à retouche abrupte.

Pour le locus, Beyssan, devant la maison, on notera la présence d'un grand nucléus (12 cm de haut), sur lequel ont été débitées de longues et fines lamelles sur un front étroit permettant des séquences de trois ou au plus quatre supports; sa mise en forme est sommaire, limitée à un seul flanc, l'autre étant constitué de cortex.

Le locus des Cerisiers livre le plus de mobilier, par ex. quinze nucléus et fragments de nucléus à lamelles, quadrangulaires plats ou subconiques tournants, débités après chauffe ou pas, une quantité considérable de fragments de fines lamelles très régulières (plus de 1500), de petit module, de section triangulaire ou trapézoïdale, en silex chauffé ou non. L'outillage compte des chanfreins (ou perçoirs à biseau) dégagés par retouche abrupte, les célèbres " perçoirs tournevis de Trets ", des becs et perçoirs, des lamelles tronquées, des burins d'angle, sept armatures géométriques : deux triangulaires à retouche rasante, plate envahissante directe et semiabrupte inverse, probablement sur éclat, et cinq trapézoïdales à retouche inverse semi-abrupte. Ces dernières sont des bitroncatures qui dans l'orientation fonctionnelle sont plus larges que longues. Elles sont majoritairement réalisées sur lames par retouche peu étendue. Leurs caractéristiques permettent de les rapprocher des bitroncatures de Trets, la Bastidonne, et plus généralement de celles d'autres sites attribués au Néolithique moyen de type La Roberte (voir ci-dessous).

En plus de la série en silex, la station a livré des pièces en obsidienne : un nucléus et trois fragments de nucléus. Il y a quelques années, ils devaient faire l'objet d'analyses afin d'en déterminer l'origine, mais ils ont disparu sans laisser de trace. Ils ont donc été examinés seulement sur photographies. Le nucléus entier montre des corniches pas ou peu abrasées et des produits d'un module relativement large, ce qui indique l'usage de la percussion indirecte. Le débitage est semi-tournant. Tous les enlèvements ou presque semblent outrepassés. En plus de ces nucléus, le locus dit des Cerisiers a livré sept fragments (éclats et fragments d'éclat, fragment de lamelle) et le locus dit Julien quatre fragments considérés comme de l'obsidienne. Ces onze objets ont fait l'objet d'une étude de provenance par l'un de nous (F.-X. Le B.) à 1'IRAMAT-CRP2A (UMR5060, université Bordeaux Montaigne et CNRS) ${ }^{(2)}$. Afin de sauvegarder l'intégrité de ces échantillons, il a été procédé à une analyse élémentaire non-destructive par EDXRF Energy Dispersive X-Ray Fluorescence Analysis (Lugliè et al., 2014).
Les caractérisations géochimiques ont été menées à l'aide du microanalyseur EDXRF SEA6000vx de chez Seiko-Hitachi équipé pour l'occasion d'un collimateur $3 \times 3 \mathrm{~mm}$. La source $\mathrm{Rh}(50 \mathrm{kV} / 1 \mathrm{~mA})$ et le détecteur SDD Vortex haute résolution autorisent, dans l'obsidienne, le dosage de dix-huit éléments majeurs, mineurs et traces. En pratique, seulement huit d'entre eux (Mn, Fe, Zn, Ga, $\mathrm{Rb}, \mathrm{Sr}, \mathrm{Y}$ et $\mathrm{Zr}$ ) sont systématiquement utilisés pour les études de provenance. Les mesures ont été effectuées en trois « points » différents sur chaque pièce, afin de tenir compte d'éventuelles hétérogénéités locales de composition ou de présence d'inclusions cristallines.

Les résultats sont présentés dans le tableau 1. Les données reportées sont les moyennes et les écarts-types des valeurs obtenues pour les trois mesures. Sur les onze échantillons analysés, neuf sont des obsidiennes. L'artefact Beyssan-Julien-3 est probablement un silex et Beyssan-Cerisiers-1 un matériau non déterminé.

Une comparaison avec les données obtenues par l'équipe de l'IRAMAT-CRP2A dans des conditions similaires sur des obsidiennes provenant des îles-sources potentielles permet d'exclure pour les obsidiennes de Beyssan une provenance des îles de Lipari, Palmarola ou Pantelleria, au bénéfice de la Sardaigne. Dans cette dernière, les sources d'obsidienne sont associées au massif volcanique du Monte Arci dans le Centre-Ouest de l'île, à l'est d'Oristano, à actuellement environ $20 \mathrm{~km}$ de la mer, où l'on reconnaît quatre types géochimiques SA, SB1, SB2 et SC (Tykot, 1997; Lugliè et al., 2006). À partir des rapports $\log (\mathrm{Y} / \mathrm{Rb})$ et $\log (\mathrm{Sr} / \mathrm{Rb})$, on constate que les objets des locus Beyssan, Cerisiers, et Beyssan, Julien, appartiennent à deux groupes distincts, ceux des obsidiennes de type SA, huit artefacts, et 1 de type SC (fig. 13).

La présence d'obsidienne sur les stations néolithiques dans le Sud de la France a depuis longtemps attiré l'attention et a toujours été la source d'interrogations, en particulier sur ses modes de diffusion (Courtin, 1973; Binder et Courtin, 1994; Vaquer, 2007; Poupeau et al., 2010). Mais, généralement, les sites ne livrent que quelques objets, moins de cinq. Seulement une dizaine de gisements en ont livré plus de dix (Binder et Courtin 1994; Binder et al., 2012), ensemble dans lequel Trets, les Terres Longues, fait figure d'exception (Pellissier, 2010). Assurément avec douze fragments (les neuf déterminés et les trois nucléus, et c'est là un minimum), Beyssan est donc l'un des gisements du Sud-Est qui en a livré le plus. La présence largement majoritaire du type SA, originaire d'une zone relativement limitée dans la partie sud du massif du Monte Arci (Conca Cannas à Masullas), fait nettement référence à ce qui est connu dans le Sud-Est de la France pour le IV millénaire, période pendant laquelle le type SA est nettement majoritaire, en particulier sur les établissements du bassin de Trets (Vaquer, 2007; Binder et al., 2012). Sur les sites qui ont livré plusieurs fragments, le type SC est rarement représenté par plus d'un exemplaire. Dans la région d'origine, ce type est cependant particulièrement facile d'accès dans la partie est du massif. Au sein des quatre types de matériaux reconnus (SA, SB1, SB2, SC), il présente en 


\begin{tabular}{|c|c|c|c|c|c|c|c|c|c|c|}
\hline Artefacts & & $\mathrm{MnO}$ & $\mathrm{Fe}_{2} \mathrm{O}_{3}$ & $\mathrm{Zn}$ & $\mathrm{Ga}$ & $\mathrm{Rb}$ & $\mathrm{Sr}$ & $\mathrm{Y}$ & $\mathrm{Zr}$ & Type \\
\hline \multirow{2}{*}{ Beyssan-Cerisiers-2 } & moyenne & 737 & 15499 & 96 & 27 & 292 & 36 & 41 & 91 & \multirow{2}{*}{ SA } \\
\hline & $\sigma$ & 37 & 1449 & 8 & 2 & 23 & 4 & 3 & 5 & \\
\hline \multirow{2}{*}{ Beyssan-Cerisiers-3 } & moyenne & 675 & 13477 & 82 & 24 & 262 & 33 & 38 & 83 & \multirow{2}{*}{ SA } \\
\hline & $\sigma$ & 20 & 401 & 1 & 2 & 5 & 1 & 0 & 4 & \\
\hline \multirow{2}{*}{ Beyssan-Cerisiers-4 } & moyenne & 737 & 16106 & 92 & 27 & 285 & 36 & 43 & 85 & \multirow{2}{*}{ SA } \\
\hline & $\sigma$ & 8 & 1468 & 2 & 1 & 11 & 1 & 3 & 4 & \\
\hline \multirow{2}{*}{ Beyssan-Cerisiers-5 } & moyenne & 694 & 13781 & 86 & 26 & 269 & 34 & 38 & 83 & \multirow{2}{*}{ SA } \\
\hline & $\sigma$ & 18 & 137 & 2 & 1 & 3 & 2 & 0 & 3 & \\
\hline \multirow{2}{*}{ Beyssan-Cerisiers- 6} & moyenne & 710 & 14540 & 95 & 26 & 281 & 35 & 40 & 89 & \multirow{2}{*}{ SA } \\
\hline & $\sigma$ & 9 & 315 & 3 & 0 & 9 & 1 & 1 & 4 & \\
\hline \multirow{2}{*}{ Beyssan-Cerisiers-7 } & moyenne & 448 & 21301 & 75 & 22 & 198 & 171 & 29 & 247 & \multirow{2}{*}{$\mathrm{SC}$} \\
\hline & $\sigma$ & 21 & 1635 & 1 & 0 & 9 & 8 & 2 & 10 & \\
\hline \multirow{2}{*}{ Beyssan-Julien-1 } & moyenne & 698 & 13878 & 87 & 27 & 274 & 34 & 41 & 90 & \multirow{2}{*}{ SA } \\
\hline & $\sigma$ & 19 & 374 & 3 & 1 & 9 & 1 & 1 & 8 & \\
\hline \multirow{2}{*}{ Beyssan-Julien-2 } & moyenne & 697 & 14219 & 85 & 26 & 268 & 34 & 37 & 82 & \multirow{2}{*}{ SA } \\
\hline & $\sigma$ & 17 & 96 & 1 & 1 & 2 & 1 & 2 & 3 & \\
\hline \multirow{2}{*}{ Beyssan-Julien-4 } & moyenne & 717 & 15339 & 89 & 27 & 285 & 35 & 42 & 88 & \multirow{2}{*}{ SA } \\
\hline & $\sigma$ & 3 & 863 & 2 & 2 & 2 & 1 & 1 & 12 & \\
\hline
\end{tabular}

$\sigma$ : écart-type (1 sigma); teneurs en $\mu \mathrm{g} / \mathrm{g}(\mathrm{ppm})$.

Tabl. 1 - Tableau des données EDXRF des obsidiennes de Beyssan (Apt, Vaucluse). Les données pour les artefacts Beyssan-Julien-3 (silex) et Beyssan-Cerisiers-1 (matériau non déterminé) ne sont pas reportées dans ce tableau.

Table 1 - Table of EDXRF data, obsidian material from Beyssan (Apt, Vaucluse). Data for artefacts Beyssan-Julien-3 (flint) and Beyssan-Cerisiers-1 (undefined) are not included in this table.

effet la plus vaste zone de disponibilité, soit dans les sites primaires à l'ouest de Pau (Sennixeddu, Scaba Crobina, Campu Serrau) soit sur des sources secondaires dans les alluvions du Rio Mogoro et du Rio Mannu au sud-est et au sud du massif (Lugliè et al., 2006). La prédominance du type SA dans le Sud-Est de la France n'est donc ni fortuite ni aléatoire et la signification de ce phénomène est encore en discussion.

Fig. 13 - Diagramme comparant les rapports $\log (\mathrm{Y} / \mathrm{Rb})$ et $\log$ $(\mathrm{Sr} / \mathrm{Rb})$ pour les 9 obsidiennes de Beyssan (Apt, Vaucluse) et celles des îles-sources méditerranéennes de Lipari, Palmarola et Sardaigne (SA, SB1, SB2 et SC). Les indications entre parenthèses $(n)$ représentent le nombre d'échantillons géologiques pour chaque source. Dans un souci de visibilité, les Pantellerites peralcalines de Balata dei Turchi et Lago di Venere ne sont pas représentées sur cette figure.

Fig. 13 - Diagram comparing the relations $\log (Y / R b)$ and $\log (\operatorname{Sr} / R b)$ for the nine obsidian artefacts from Beyssan (Apt, Vaucluse) and those from Mediterranean source-islands of Lipari, Palmarola and Sardinia (SA, SB1, SB2 and SC). Indications between backers (n) show the number of geological samples for each source. For a better visibility, peralacline pantellerites from Balata dei Turchi and Lago de Venere are not shown in this figure.
L'industrie de pierre polie comporte des lames et des billes. Les douze billes entières et les quatre fragments sont en matériau de différentes natures mais principalement en calcaire, leur diamètre varie de 2,3 à $3,5 \mathrm{~cm}$. Un objet plus petit $(1,77 \mathrm{~cm}$ de diamètre $)$ unique par ces dimensions et sa régularité pourrait être un biscayen. Ces objets ont depuis longtemps attiré l'attention car ils paraissent relativement fréquents dans des sites

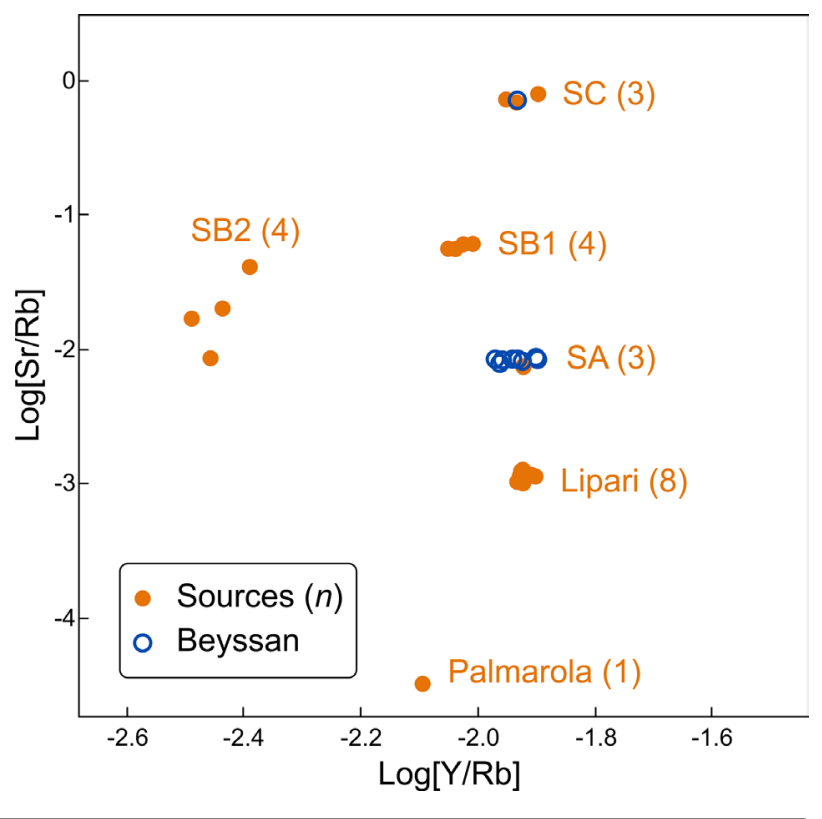


du Néolithique moyen (Cotte, 1916; Courtin, 1974). Cependant, ils ne se retrouvent pas partout; le dernier décompte en dénombre 391 sur 95 sites, souvent des fouilles anciennes ou des ramassages de surface et régulièrement associées au monde des morts (Thirault, 2006).

Les lames de haches polies sont de différents modules et différentes matières premières duranciennes ou alpines : métabasite à glaucophane et éclogite à grenat pour les plus grandes (11 et $12 \mathrm{~cm})$. Les plus petites $(4 \mathrm{~cm}$ pour les deux entières) sont probablement en différentes natures de jadéite de couleur claire.

\section{Une troisième stèle?}

Pour être complet, il convient de mentionner l'existence d'une probable troisième stèle sur le locus de Beyssan Nord-Ouest. Elle gisait en bordure de parcelle, probablement à la suite d'un épierrement. Il s'agissait d'une dalle subtriangulaire de calcaire oligocène d'environ $50 \mathrm{~cm}$ de haut et $35 \mathrm{~cm}$ de large. Un peu plus grande donc que les deux autres stèles. Cette troisième stèle ne porte ni décor ni traitement de surface. Il pouvait s'agir soit d'une stèle totalement aniconique soit d'un matériau destiné à une autre fabrication, ce que laissent supposer le sommet concave et l'aménagement des bords. L'objet a disparu sans avoir été étudié en détail. Il attire néanmoins l'attention sur le fait qu'à côté des stèles décorées, il pouvait exister des monuments sans décor évident et qui n'ont probablement pas toujours été reconnus à l'occasion des recherches anciennes.

Cette association de stèles décorées et de stèles aniconiques a déjà été plusieurs fois signalée principalement pour la période plus récente du Néolithique final. Ainsi, en Languedoc, sur les sites Ferrières gardois de Courion et de Montaïon. Sur ce dernier, plusieurs dalles aniconiques étaient associées à la stèle anthropomorphe, l'ensemble étant implanté sur un "monument», probablement en forme de tertre, érigé à proximité d'un habitat (Gutherz et Jallot, 1987; Gutherz et al., 1998). De telles associations ont été également signalées sur le versant italien des Alpes (Fedele, 2008; Fedele et Fossati, 2012a). Ce constat conduit à poser la question de la mise en évidence des sites ne comportant que des stèles non sculptées où ne comportant qu'un décor minimal. Celles-ci sont en effet nombreuses dans la fin du Néolithique mais également dans le Néolithique moyen (Jallot, 1998; Rodriguez 1998) et ont été généralement assimilées à des menhirs proto-anthropomorphes; les sites corses de Renaghju et i Stantari sur le plateau de Cauria montrent qu'elles constituent parfois des ensembles numériquement importants (D’Anna et al., 2007; D’Anna, 2015b). Ces observations confirment que la limite entre les notions de menhirs et de statues-menhirs n'est pas toujours très nette et facile à déterminer, ce qui a fait l'objet de différentes discussions (Voruz, 1990 et 1992; Jallot 2003; Cassen, 2009). C'est dans la perspective d'une telle étude globale du phénomène que doivent être proposées les interprétations (voir ci-dessous).

\section{Chronologie}

Les stèles provençales à décors de chevrons sont relativement bien datées. Elles ont toujours été rattachées au Néolithique moyen (Arnal, 1970). Ainsi, elles ont été attribuées au Chasséen (Arnal, 1976a et b), au Chasséen B (Arnal et Arnal, 1984), au Lagozzien de Trets (Escalon de Fonton, 1962; Gagnière et Granier, 1963 et 1976; Landau 1977) et surtout au Chasséen récent ou évolué (D’Anna, 1977 et 2015a; Sauzade, 1983 ; D'Anna et al., 1997; Guilaine 1998 et 2003). Ce qui veut dire la même chose. Dans un contexte de réflexion en évolution sur la fin du Néolithique moyen, elles ont été rapportées à une période entre 3600 et 3300 av. J.-C., et donc antérieures aux autres manifestations de la statuaire de la fin du Néolithique (D’Anna, 2002a et b). Les dernières découvertes, Château Blanc, Goult et donc Gargas, confirment ce qui avait été proposé : les stèles à chevrons n'appartiennent pas au Néolithique final comme les statues-menhirs du Rouergue et du Languedoc, mais s'inscrivent dans la fin du Néolithique moyen, dans le contexte chronoculturel de la première partie du $\mathrm{IV}^{\mathrm{e}}$ millénaire, entre 3800 et 3500 av. J.-C. Cette période fait encore débat et reste en cours de caractérisation (Van Willigen et al., 2012; Lepère, 2012; Gernigon, 2014). Avec la découverte de Gargas qui n'est donc pas sans rappeler celles de la Bastidonne à Trets et du Frigouret à Villecroze (Gagnière, 1954, p. 126), cela ne fait plus de doute. À Beyssan, le contexte très homogène indique bien une période entre environ 3800 et 3500 avant notre ère, Chasséen récent ou Néolithique moyen de type La Roberte selon les auteurs. On retrouve ici, d'une certaine manière, l'attribution au « Néolithique de type Trets » dont les relations avec le Chasséen ont été longtemps discutées (Escalon de Fonton, 1968 et 1971; Escalon de Fonton et Palun 1955; Courtin, 1974; D’Anna et Mills, 1981) et qui a été depuis requalifié et mis en perspective (Binder, 1991; Van Willigen et al., 2012). Comme on l'a constaté dans les descriptions, les points communs entre les stèles de Beyssan et celles de Trets sont nombreux, en particulier pour ce qui concerne la stèle 1 de Beyssan : forme nettement triangulaire (la Bastidonne 5 et 6 ), absence des éléments anatomiques yeux-nez, morphologie des gravures, absence de relief (la Bastidonne 4, 5 et 6), utilisation de cinabre.

\section{Discussion}

Si la datation des stèles provençales peut être considérée comme globalement acquise, il n'en n'est pas de même pour ce qui concerne leur contexte précis. Afin de mettre la découverte de Gargas en perspective, il faut encore une fois rappeler les données relatives au site de la Bastidonne à Trets comme cela a déjà été fait en détail (D’Anna al., 2004).

Le site a été découvert en 1870, mais l'inventeur J. Maneille n'a pas publié ses trouvailles; elles l'ont seulement été plusieurs années après au début du $\mathrm{Xx}^{\mathrm{e}}$ siècle. Le gisement avait alors été partiellement détruit et la collection dispersée. Il est donc difficile d'avoir une vision précise du contexte et de l'organisation du site qui semble 
relativement complexe. Toutes les publications anciennes sont donc de « deuxième main » (voire de troisième!) et généralement sans illustration; elles s'accordent pour considérer que les fragments de stèles étaient associés à des tombes à incinération et à d'autres dalles non décorées; mais les descriptions divergent sur la position des stèles : entre les sépultures, directement associées aux petits coffres dans lesquels elles étaient placées ou encore, brisées, constituant ces coffres et les recouvrant (Chaillan, 1893 ; Fournier et Répelin, 1901 ; Cotte, 1905 ; GerinRicard, 1899 et 1910; Gerin-Ricard et Arnaud d'Agnel, 1907). Il est donc possible que les fragments de stèles aient été là en réemploi ce « qui ne résout nullement l'origine de ces figurations schématiques » (Courtin, 1974). Une seule de ces publications distingue trois secteurs différents : deux zones de sépultures et une d'habitat (Cotte, 1905). Les recherches du $\mathrm{XX}^{\mathrm{e}} \mathrm{s}$. sont restées limitées (Escalon de Fonton et Palun, 1955 ; Escalon de Fonton, 1962; D'Anna, 1977; D’Anna et Mills, 1981). Elles permettent cependant de confirmer que le site de la Bastidonne recelait au moins deux secteurs avec des sépultures de natures différentes et une troisième zone n'en comportant aucune.

Le premier locus, vers le sud-est, est celui des sépultures à inhumation. La dernière fouillée et publiée, sur la bordure sud-ouest de la partie supérieure du plateau montre des dépôts multiples répartis en trois niveaux séparés par des dallettes. Le mobilier peu abondant peutêtre attribué aux dernières phases du Néolithique moyen. Aucune recherche plus récente n'a livré de fragment de stèle dans ce secteur, mais seulement des petits fragments d'os et une fosse avec les squelettes de trois grands chiens et ne contenant que deux fragments de lamelles (D'Anna, 1978, rapport de fouille, inédit). Il n’est donc pas impossible que ce secteur recèle d'autres sépultures.

Le deuxième locus, vers le nord-ouest du plateau, plus proche de la ferme, est celui des sépultures à incinération dont aucune n'a été observée depuis la découverte de Maneille; le secteur livrait encore récemment, dans la partie défrichée au XIX ${ }^{\mathrm{e}}$ siècle, des petits fragments de stèles, des fragments d'os brûlés, de l'industrie lithique comportant des fragments de lamelles et des armatures trapézoïdales à retouches abruptes qui constituent une série originale par leur nombre cumulé.

Il est utile d'examiner ici cette série en détail. Elle comporte quarante-trois objets dont vingt et un complets; trente-sept peuvent être caractérisés d'un point de vue typologique. Toutes ces armatures sont brûlées à divers stades et la matière première n'est pas identifiable. On peut déterminer la nature des supports d'origine dans quarante cas : sept sont réalisées sur des lames à deux pans, deux sur des lamelles à trois pans, dix-huit sur des lames à trois pans et à code opératoire 212', deux sur des lames à trois pans et à code opératoire 123/321, huit sur des lames à trois pans et à code opératoire indéterminé et trois sur des fragments de lames indéterminés. Elles se répartissent en trois classes typologiques principales (Binder, 1987) : sept sont des « bitroncatures géométriques à troncatures directes », elles sont toutes trapézoïdales; cinq sont des « bitroncatures géométriques à troncatures inverses »; trois sont trapézoïdale et deux triangulaires; enfin, vingt-quatre sont des « bitroncatures géométriques à troncatures inverses et à retouches directes rasantes » dont vingt-deux sont trapézoïdales et une est triangulaire; dans tous les cas, les retouches directes sont courtes. On note qu'une des armatures est un trapèze symétrique à deux bords concaves et n'a donc pas pu être classée dans la liste type. Du point de vue morphométrique le rapport longueur (hauteur)/largeur ( $\mathrm{du}$ 《 tranchant ») montre une majorité d'éléments plus larges que longs. Ces bitroncatures géométriques sont pour la totalité réalisées sur lames et sont en majorité des trapèzes. L'ensemble se caractérise par une représentation importante du code opératoire $212^{\prime}$ ce qui est une caractéristique des phases récentes du Néolithique moyen (Binder, 1984), ce qui semble être également le cas de la forme plus large que longue. Les meilleures comparaisons pour cette série de Trets et donc pour celle de Gargas se retrouvent dans la structure 10 de la Prairie à Chabrillan (Saintot et Brochier, 2002), la structure 8048 de la Ramière (Léa, 2004), les couches 7 et 6 de la grotte de l'Église Supérieure (Courtin, 1974; Léa, Linton, Gassin, 2009), les couches 18 et 19 de la Baume Fontbrégoua (Binder, 1987) ou encore sur le site de Lattes (Léa, 2004). Ce type d'armature est donc principalement associé au Néolithique moyen de type La Roberte et n'est présent ni dans le Chasséen, ni dans le Néolithique moyen de type Mourre de la Barque (van Willigen et al. 2012).

Enfin, Le troisième locus de la Bastidonne est situé au sud, en contrebas du plateau, au pied des petites falaises qui limitent le plateau portant les locus 1 et 2 , il a seulement livré de l'industrie lithique, mais jamais ni sépultures, ni stèles.

Il n'est pas inutile de rappeler que le troisième type de sépulture reconnu à Trets, en fosses-silos, ne provient pas de la Bastidonne (Courtin, 1974) et que c'est le mobilier de ces dernières qui a été sérié et rattaché au Néolithique moyen de type La Roberte (Van Willigen et al., 2012).

Si l'on replace le site de la Bastidonne dans le contexte de l'ensemble des stations reconnues dans le bassin de Trets (D’Anna et Mills, 1981) on remarque que, du point de vue topographique et géomorphologique, il est dans le prolongement de l'établissement de Sainte-Catherine, avec lequel la discontinuité est d'environ de $200 \mathrm{~m}$. Les industries lithiques de Sainte-Catherine, longtemps considérées comme caractéristiques du Néolithique de type Trets ont été remises en perspective (Binder, 1991), et l'évolution des connaissances permet bien de les placer dans le Néolithique moyen de type La Roberte. Il est donc possible de considérer la Bastidonne comme représentant un locus particulier en limite sud-est de la zone principale d'un vaste établissement. Une telle extension est cohérente avec ce qui a été reconnu dans la vallée du Rhône (Beeching et al., 2000) ou le Toulousain (Vaquer, 1991) et à Gargas.

C'est en grande partie par comparaison avec la Bastidonne que le contexte des deux stèles de la Lombarde (ou Lombardie selon les auteurs) à Puyvert a été considéré comme également en lien avec des sépultures, ce que la présence de fragments d'os humains, d'une grande lame de hache polie et de plusieurs petites dalles suggère en effet (Gagnière et Granier 1963, 1967 et 1976). Si la présence de quelques fragments d'os brulés pose la question 
de la présence d'incinérations, les sondages n'ont livré aucun résultat (Courtin, 1974, p. 146).

La fouille de la nécropole de Château Blanc, en 1995, sur le tracé du TGV Méditerranée, a apporté la confirmation de l'association des stèles provençales avec le domaine funéraire. Le site de Ventabren comporte cinq tertres de terre ceinturée de pierres qui livrent trois phases distinctes de fonctionnement. C'est à la plus ancienne qu'ils couvrent une sépulture centrale en fosse aménagée de dalles ou non. Autant que l'on puisse en juger du fait des problèmes taphonomiques, ce sont des sépultures individuelles ou doubles. Elles possèdent, en périphérie des « logettes », petit coffre en pierre, qui contenaient les stèles. Celles-ci ne sont dont pas directement dans ou sur les sépultures mais dans des structures associées, implantées à quelques mètres (Hasler, 1998; Hasler et al., 1998 et 2002). Elles n'étaient pas plantées mais appuyées contre le parement interne de la logette. La situation stratigraphique indique clairement une position antérieure à l'élévation du dolmen du tertre IV (phase trois du site) et l'utilisation des stèles pendant le IV e millénaire dans un contexte de sépultures en fosse. Il n'y a aucun indice d'incinération. Malheureusement, les datations radiométriques, en contradiction avec la stratigraphie, ne sont pas utilisables.

Enfin, dans la vallée du Calavon, les deux stèles de l'Ubac à Goult confirment une utilisation avant l'érection $\mathrm{du}$ dolmen, mais ne livrent aucune indication sur leur implantation d'origine et leur contexte initial (Sauzade et al., 2003; Bizot et al., 2004; Sauzade, 2012). C'est seulement par comparaison et cohérence avec Château Blanc que l'on peut déduire qu'elles étaient associées à un ensemble funéraire dont aucun vestige n'est visible !

L'ensemble de ces éléments indique de manière relativement crédible l'association des stèles provençales avec le domaine funéraire. Mais les informations, si elles sont fiables pour ce qui concerne la chronologie, sont partiellement contradictoires pour ce qui concerne la nature précise des contextes, en particulier pour ce qui est de l'association avec des sépultures à incinération. S'il y a eu incinération, au moins à Trets, on ne sait pas de quoi ! Une telle pratique, faisant intervenir l'incinération est maintenant bien connue pour le Néolithique moyen de type Chassey avec la fouille des Bagnoles à L'Isle-sur-laSorgues (fouilles en cours dirigées par S. Van Willigen) mais elle ne l'est pas pour des phases plus récentes du Néolithique moyen. Par ailleurs, il convient de noter que l'association des stèles avec des sépultures en fosse, aménagée ou non, de la fin du Néolithique moyen, ce qui est avérée à Château Blanc, n’est pas un phénomène généralisé. Au contraire, si les sépultures de cette période sont de mieux en mieux connues en particulier par des fouilles d'archéologie préventive réalisées sur de vastes superficies, elles ne montrent jamais une telle association, tant en Provence qu'en vallée du Rhône et Languedoc (Schmitt, 2015). On peut mentionner, par exemple, à $8 \mathrm{~km}$ de Gargas, le site des Martins à Roussillon qui a livré quatre sépultures attribuables au Néolithique moyen de type La Roberte (D'Anna, 1993; Van Willigen et al., 2012) et sur lequel aucune stèle n'a été identifiée.
Il semblerait donc que les stèles provençales constituent un phénomène particulier qui pourrait être lié à des sites d'un type « intermédiaire » qui n'a pas été correctement caractérisé. Cela a déjà été suggéré : « les stèles provençales ne seraient elles pas associées à un autre type de site, tels que sanctuaire, lieux de cultes » (Sauzade, 1987).

De tels sites peuvent être nommés de différentes manière : site cérémoniel, site cultuel, sanctuaire, centre rituel, espace sacré (Fedele, 2004 et 2008). Ils ont été reconnus aussi bien dans des périodes un peu plus anciennes qu'un peu plus récentes que Beyssan. Ils sont implantés dans les milieux les plus divers et tous montrent de longues occupations. Ils sont principalement matérialisés par des pierres dressées, parfois de hauteur modeste parfois très grandes, sculptées ou non. À la suite de plusieurs fouilles relativement étendues, leur connaissance est en pleine progression. Pour les périodes un peu plus anciennes du Néolithique moyen, on mentionnera par exemple les sites suisses de Derrière la Croix à SaintAubin (Wütrich, 2003), Bevaix (Grau Bitterli et FierzDayer, 2011), Lutry et Yverdon (Voruz, 1990 et 1992). En milieu insulaire, les alignements de Cauria sont évidemment des cas exemplaires de sites cérémoniels du Néolithique moyen réutilisés à l'âge du Bronze (D'Anna et al., 2007; D’Anna, 2014 et 2015b). Pour les périodes un peu plus récentes de la fin du Néolithique, ces sites cérémoniels intégrant des stèles sont nombreux dans les Alpes en particulier dans le Valcamonica. Ainsi à Ossimo, Pat (Poggiani-Keller, 2013) où une série de monolithes alignés relient deux aires, l'une de tumulus et l'autre de cercles. Le site est occupé pendant une longue durée et la première phase est située vers 3700-3500 av. J.-C. De même à Ossimo-Bormo, un alignement avec au moins trois stèles gravées et une stèle aniconique connaît une première phase d'occupation au début du $\mathrm{III}^{\mathrm{e}}$ millénaire (Fedele, 2004; Fedele et Fossati, 2012b). On peut également mentionner les niveaux préfunéraires de Sion et Aoste (Gallay, 1995 ; Mezzena, 1998; Corboud, 2009).

Dans les premières phases d'utilisation il n'y a aucun lien direct entre ces monuments et le domaine funéraire. Les sépultures semblent s'installer dans un deuxième temps. Dans le Midi de la France, le contexte des statues-menhirs est mal connu, mais quelques cas montrent clairement l'existence d'espaces cérémoniels dont le meilleur exemple est Montaïon, site sur lequel une stèle anthropomorphe et plusieurs dalles aniconiques étaient implantées sur un "monument » érigé à proximité d'un habitat (Gutherz et Jallot, 1987).

On retrouve donc ici l'ensemble de la question des sites de pierres dressées qui pendant tout le Néolithique et l'âge du Bronze, au moins dans certaines régions, viennent s'intercaler entre les habitats et les nécropoles. Au Néolithique moyen, les sépultures sont à proximité ou dans les habitats alors que les sites cérémoniels sont isolés. Au Néolithique final, il y a une sorte d'inversion des associations avec les sépultures qui se « rapprochent» des sites cérémoniels, alors que les habitats vont prendre une autre dimension, en intégrant à côté des activités domestiques des fonctions symboliques. Les sites cérémoniels 
montrent de longues occupations, avec bris des statues, réemplois et réaménagements. Dans cette perspective, rattacher les stèles (et statues-menhirs) uniquement au domaine funéraire serait donc réducteur. À la fin du Néolithique moyen, entre 3900 et 3600 avant notre ère de tels sites cérémoniels pourraient constituer des locus spécifiques à proximité ou en marge de vastes établissements comme cela est le cas, en quelque sorte, à Château Blanc, à la Bastidonne et apparemment à Beyssan. Leur relatif isolement et la discrétion des vestiges en dehors des stèles pourraient expliquer la difficulté à les reconnaître.

\section{CONCLUSION}

L es stèles de Beyssan apportent des informations importantes, en particulier pour ce qui est de leur chronologie pendant la première partie du IV e millénaire (3800-3600 avant notre ère), dans un Chasséen récent ou Néolithique moyen de type La Roberte, selon les terminologies. D'une certaine manière, elles contribuent à caractériser cette phase. Elles proviennent d'un locus particulier d'un très vaste établissement.

Les stèles néolithiques de Provence sont aujourd'hui nombreuses de plus d'une cinquantaine d'exemplaires qui ont été en grande partie visibles lors de la remarquable exposition du musée Calvet d'Avignon dans le cadre du centenaire de la Société préhistorique française (D'Anna et al., 2004). Il serait heureux qu'elles puissent être ainsi accessibles de manière permanente dans le cadre d'un musée qui les accueillerait et les présenterait à l'image de ce qui a été fait à Rodez pour les statues menhirs du groupe rouergat (Philippon, 2002).

\section{NOTES}

(1) Nous tenons à remercier la famille Santi, propriétaire du terrain, Maxence Bailly, Jacques Élie Brochier, Dominique Commelin, René Coutel, Christine Durand, Daniel Helmer, André Kaufmann, Olivier Lemercier, Pierre Pétrequin, Guillaume Robin, Florian Soula, Samuel Van Willigen pour leurs contributions, aides, avis, observations, conseils, éléments de réflexions et remarques.

(2) Les analyses EDXRF n'auraient pas été possibles sans le soutien du conseil régional d'Aquitaine à travers le projet « La radioluminescence des feldspaths : un nouvel outil de datation des gisements archéologiques et des séquences quaternaires d'Aquitaine ». Elles ont été également soutenues par le labex « Sciences archéologiques » de Bordeaux, programme financé par l'ANR ( $n^{\circ}$ ANR-10LABX-52).

\section{RÉFÉRENCES BIBLIOGRAPHIQUES}

Anati E., Varela Gomes M. (2013) - The Züschen I Megalithic Monument (Kassel, Hessen) and its Engravings: Animal Traction, Ploughs, Carts and Wagons in Neolithic Europe, Lisbonne, Istituto de Arqueologia e Paleociências, 182 p.

Arnal G.-B., Arnal J., Lassure C., Pauze B. (1986) - Le dolmen du Pouget (Hérault) et son contexte archéologique, Lodève, Centre de recherches archéologiques du haut Languedoc (Mémoire du CRAHL, 4), $81 \mathrm{p}$.

Arnal J. (1970) - Le mystère des statues menhirs du Midi de la France, Archéologia, 36, p. 45-53.

Arnal J. (1976a) - Les statues-menhirs, hommes et dieux, Toulouse, Hespérides (Archéologie, horizons neufs), 239 p.

Arnal J. (1976b) - L'art protohistorique : les statues-menhirs de France, in J. Guilaine (dir.), La Préhistoire française, II. Les civilisations néolithiques et protohistoriques de la France, Paris, Éd. du CNRS, p. 211-221.

Arnal J., Arnal S. (1984) - Chronologie interne des statuesmenhirs en Europe occidentale, Cahiers ligures de Préhistoire et de Protohistoire, 1, p. 157-196.

Arnaud d'Agnel G. (1901) - Un groupe de dix stations préhistoriques sur le plateau des Claparèdes (Vaucluse), Répertoire des travaux de la Société statistique de Marseille, 45, p. 415-431.

Baldoni E., De Waele J., Galli E., Messina M., Onac B. P., Sanna L., Sauro F., Villani M. (2013) - Mineralogy and Speleogenesis of the Corona 'E Sa Craba Quartzite Cave (Southwest Sardinia), Proceedings of the 27th inter- national ACM conference on International conference on supercomputing, New York, ACM Press, p. 437-442.

Beeching A., Berger J.-F., Brochier J.-L., Ferber F., Helmer D., Sidi Maamar H. (2000) - Chasséens : agriculteurs ou éleveurs, sédentaires ou nomades? Quels types de milieux, d'économies et de sociétés?, in M. Leduc, N. Valdeyron et J. Vaquer (dir.), Sociétés et espaces, actes des III $^{\text {es }}$ Rencontres méridionales de Préhistoire récente (Toulouse, 6-7 novembre 1998), Toulouse, Archives d'écologie préhistorique, p. 59-80.

BINDER D. (1984) - Systèmes de débitage laminaire par pression : exemples chasséens provençaux, in J. Tixier, M.-L. Inizan et H. Roche (dir.), Préhistoire de la pierre taillée, 2. Économie du débitage laminaire, Paris, Cercle de recherches et d'études préhistoriques, p. 71-84.

Binder D. (1987) - Le Néolithique ancien provençal : typologie et technologie des outillages lithiques, Paris, CNRS éd. (Supplément à Gallia Préhistoire, 24), 209 p.

BINDER D. (1991) - Facteurs de variabilité des outillages lithiques chasséens dans le Sud-Est de la France, in A. Beeching, D. Binder, J.-C. Blanchet et al. (dir.), Identité du Chasséen, actes du colloque international (Nemours, 1989), Nemours, APRAIF (Mémoire du musée de Préhistoire d'Île-de-France, 4), p. 261-272.

Binder D. (1998) - Silex blond et complexité des assemblages lithiques dans le Néolithique liguro-provençal, in A. D'Anna et D. Binder (dir.), Production et identité culturelle, actes des $\mathrm{II}^{\mathrm{es}}$ Rencontres méridionales de Préhistoire récente (Arles, 8-9 novembre 1996), Antibes, APDCA, p. 111-128. 
Binder D., Courtin J. (1994) - Un point sur la circulation de l'obsidienne dans le domaine provençal, Gallia Préhistoire, 36, p. 310-322.

Binder D., Gratuze B., Vaquer J. (2012) - La circulation de l'obsidienne dans le Sud de la France au Néolithique, in M. Borrell, F. Borrell Tena, J. Bosch Argilagós et al. (dir.), Actes, Xarxes al Neolitic : congrès internacional, Gavà, Museu de Gavà (Rubricatum, 5), p. 189-199.

Bishop A. C., Hamilton W. R., Woolley A. R. (2005) Guide des minéraux, roches et fossiles, Paris, Delachaux et Niestlé (Les guides du naturaliste), 336 p.

Bizot B., Michel J., Sauzade G. (2004) - Dépôts funéraires et sédimentation dans le dolmen de l'Ubac à Goult, in J. Buisson-Catil, A. Guilcher, C. Hussy et al. (dir.), Vaucluse préhistorique : le territoire, les hommes, les cultures et les sites, Le Pontet, Barthélémy, p. 238-244.

Bretagne P., D’Anna A. (1988) - Bonnieux : Les Fabrys, habitat de plaine, Néolithique final-Chalcolithique, Notes d'information et de liaison Provence-Alpes-Côte d'Azur. Direction des antiquités historiques, 5, p. 163-166.

Brochier J.É., Livache M. (2004) - Les traces des derniers chasseurs-cueilleurs, in J. Buisson-Catil, A. Guilcher, C. Hussy et al. (dir.), Vaucluse préhistorique : le territoire, les hommes, les cultures et les sites, Le Pontet, Barthélémy, p. 111-126.

CASSEN S. (2009) - Exercice de stèle : une archéologie des pierres dressées. Réflexion autour des menhirs de Carnac, Paris, Errance, 158 p.

Chaillan M. (1893) - Recherches archéologiques et historiques sur Trets et sa vallée, Lafitte Reprints 1975, Marseille, Marpon et Flammarion, 234 p.

Corboud P. (2009) - Les stèles anthropomorphes de la nécropole néolithique du Petit Chasseur à Sion (Valais, Suisse), Bulletin d'études préhistoriques et archéologiques alpines, 20, p. 1-89.

Corseaux S., Courtin J., D’Anna A., Mosset C. (1984) Une stèle anthropomorphe néolithique à Villeneuve (Alpes de Haute-Provence), Bulletin de la Société préhistorique française, 81, 4, p. 113-116.

Cotte C. (1905) - Recherches aux environs de Trets (Bouchesdu-Rhône), L'Homme préhistorique, 3, p. 308-319.

Coтte C. (1916) - Discussion sur les billes en pierre polie, Bulletin de la Société préhistorique française, 13, p. 251-254.

Cotte V. (1924) - Documents sur la Préhistoire de Provence. Troisième partie : stations néolithiques et protohistoriques, Aix-en-Provence, A. Dragon, 178 p.

Courtin J. (1973) - Le problème de l'obsidienne dans le Néolithique du Midi de la France, in Hommage à Fernand Benoit, I, Bordighera, Institut international d'études ligures (Rivista di studi liguri), p. 93-109.

Courtin J. (1974) - Le Néolithique de la Provence, Paris, Klincksieck (Société préhistorique française, Mémoire 11), 360 p.

D'Anna A. (1977) - Les Statues-menhirs et stèles anthropomorphes du Midi méditerranéen, Paris, CNRS, 277 p.

D'Anna A (1993) - L'habitat de plein air en Provence : recherches récentes, in J.-C. Blanchet, A. Bulard, C. Constantin et al. (dir.), Le Néolithique au quotidien, actes du $16^{\mathrm{e}} \mathrm{Col}-$ loque interrégional sur le Néolithique (Paris, 5-6 novembre 1989), Paris, Maison des sciences de l'homme (Documents d'archéologie française, 39), p. 72-83.

D'AnnA A (2002a) - Les statues-menhirs en Europe à la fin du Néolithique et au début de l'âge du Bronze, in A. Philippon (dir.), Statues-menhirs : des énigmes de pierre venues $d u$ fond des âges, Rodez, Éd. du Rouergue, p. 196-223.

D'Anna A. (2002b) - Les sculptures de la fin du Néolithique en Méditerranée occidentale, Documents d'archéologie méridionale, 25, p. 247-256.

D'Anna A. (2014) - Le plateau de Cauria (Sartène, Corse-duSud), quinze années de recherches archéologiques, un bilan d'étape, in I. Sénépart, F. Leandri, J. Cauliez et al. (dir.), Chronologie de la Préhistoire récente dans le Sud de la France : acquis 1992-2012, actualité de la recherche, actes des $10^{\text {es }}$ Rencontres méridionales de Préhistoire récente (Porticcio, 18-20 octobre 2012), Toulouse, Archives d'écologie préhistorique, p. 309-322.

D'Anna A (2015a) - D'un colloque de Saint-Pons à l'autre, quinze ans de recherche sur les pierres dressées et la statuaire néolithique, in G. Rodriguez et H. Marchesi (dir.), Statues menhirs et pierres levées du Néolithique à aujourd'hui, actes du $3^{\text {e }}$ Colloque international sur la statuaire mégalithique (Saint-Pons-de-Thomières, 12-16 septembre 2012), Montpellier, service régional de l'Archéologie de LanguedocRousillon; Saint-Pons-de-Thomières, Groupe archéologique Saint-Ponais, p. 15-26.

D'Anna A (2015b) - Les pierres dressées et les statuesmenhirs de Corse : contexte, chronologie, origines, in G. Rodriguez et H. Marchesi (dir.), Statues menhirs et pierres levées du Néolithique à aujourd'hui, actes du $3^{\text {e }}$ Colloque international sur la statuaire mégalithique (Saint-Pons-de-Thomières, 12-16 septembre 2012), Montpellier, service régional de l'Archéologie de LanguedocRousillon; Saint-Pons-de-Thomières, Groupe archéologique Saint-Ponais, p. 313-327.

D’Anna A., Courtin J., Coutel R., Müller A. (1989) Habitats perchés et enceintes du Néolithique final et Chalcolithique dans le Luberon central (Vaucluse), in A. D'Anna et X. Gutherz (dir.), Enceintes, habitats ceinturés, sites perchés du Néolithique au Bronze ancien dans le Sud de la France et les régions voisines, actes de la table-ronde (Lattes et Aix-enProvence, 15-18 avril 1987), Aix-en-Provence, Association pour le développement de l'archéologie en PACA; Montpellier, Société languedocienne de Préhistoire (Mémoire de la Société languedocienne de Préhistoire, 2), p. 165-193.

D’Anna A., Guendon J.-L., Orsini J.-B., Pinet L., TraMONI P. (2007) - Le plateau de Cauria à l'âge du Bronze : de la lecture évènementielle à l'approche anthropologique, in J. Évin (dir.), Un siècle de construction du discours scientifique en Préhistoire, actes du $26^{\mathrm{e}}$ Congrès préhistorique de France, congrès du centenaire de la Société préhistorique frnçaise (Avignon, 26-24 novembre 2000), Paris, Société préhistorique française, vol. 2, p. 331-346.

D'Anna A., Gutherz X., Jallot L. (1997a) - L'art mégalithique dans le Midi de la France. Les stèles anthropomorphes et les statues-menhirs néolithiques, in J. L'Helgouach, C.-T. Le Roux et J. Lecornec (dir.), Art et symboles du mégalithisme européen, actes du $2^{\mathrm{e}}$ Colloque international sur l'art mégalithique (Nantes, juin 1995), Rennes, Pôle éditorial 
archéologique de l'Ouest (Supplément à la Revue archéologique de l'Ouest, 8), p. 179-193.

D'Anna A., Lazard-Dhollande N., Lemercier O. (1997b) - Une stèle anthropomorphe néolithique trouvée près de Cavaillon (Vaucluse) acquise par le musée des Antiquités nationales, Antiquités nationales, 29, p. 21-26.

D'Anna A., Mills N. T. W. (1981) - L'occupation néolithique du bassin de Trets (Bouches-du-Rhône) France, Bulletin archéologique de Provence, 8, 4, p. 3-46.

D’Anna A., Renault S., Guendon J.-L., Masse J.-P., Pinet L., Walter P. (2004) - Stèles anthropomorphes néolithiques de Provence : catalogue du musée Calvet d'Avignon, Avignon, établissement public Calvet, $96 \mathrm{p}$

Dawson P. (1972) - The Vibrational Spectrum of $\alpha$-mercuric Sulphide, Spectrochimica Acta. Part A: Molecular Spectroscopy, 28, 12, p. 2305-2310.

Duda R., Rejl L., Slivka D., Bariand P. (1986) - La Grande encyclopédie des minéraux, Paris, Gründ (Grandes encyclopédies), $520 \mathrm{p}$.

Escalon de Fonton M. (1962) - Les stèles de Trets, Antiquités nationales et internationales, 3, 1-2, p. 8-12.

Escalon de Fonton M. (1968) - Préhistoire de la basse Provence occidentale. Tome 1, Martigues, Syndicat d'inititative et office du tourisme de la région de Martigues, $71 \mathrm{p}$.

Escalon de Fonton M. (1971) - Le Lagozien, in J. Guilaine (dir.), Les Civilisations néolithiques du Midi de la France, actes du colloque (Narbonne, 15-17 février 1970), Carcassonne, Laboratoire de Préhistoire et de palethnologie, p. 56-58.

Escalon de Fonton M., Palun Y. (1955) - Le Lagozien de Trets (Bouches-du-Rhône) : une sépulture en fosse à la Bastidonne, Cahiers rhodaniens, 2, p. 9-16.

Fedele F. G. (2004) - Monoliths and Human Skeletal Remains: Ritual Manipulation at the Anvòia Ceremonial Site, Ossimo (Val Camonica, Italy), in S. Casini et A. E. Fossati (dir.), Le pietre degli dei: statue-stele dell'Età del Rame in Europa, Bergame, Civico Museo archeologico (Notizie archeologiche bergomensi, 12), p. 49-66.

Fedele F. G. (2008) - Statue-Menhirs, Human Remains and Mana at the Ossimo 'Anvòia' Ceremonial Site, Val Camonica, Journal of Mediterranean Archaeology, 21, 1, p. 57-79.

Fedele F. G., Fossati A. E. (2012a) - L'area cerimoniale di Anvòia a Ossimo, Valcamonica: i monoliti simbolici e il loro contesto, in Istituto italiano di preistoria e protostoria (dir.), L'arte preistorica in Italia, actes de la XLII ${ }^{\mathrm{e}}$ Riunione scientifica dell'Istituto di Preistoria e Protostoria, Trento (Riva del Garda, Val Camonica, 9-13 octobre 2007), Trente, Museo tridentino di Scienze naturali (Preistoria Alpina. Rendiconti della Società cultura preistorica tridentina, 46-2), vol. 2, p. 189-199.

Fedele F. G., Fossati A. E. (2012b) - Massi, menhir e stele: alcuni particolari monoliti simbolici dal sito cerimoniale calcolitico di Anvòia a Ossimo (Valcamonica), in Istituto italiano di preistoria e protostoria (dir.), L'arte preistorica in Italia, actes de la XLII ${ }^{\mathrm{e}}$ Riunione scientifica dell'Istituto di Preistoria e Protostoria, Trento (Riva del Garda, Val Camonica, 9-13 octobre 2007), Trente, Museo tridentino di Scienze naturali (Preistoria Alpina. Rendiconti della Società cultura preistorica tridentina, 46-2), vol. 2, p. 281-282.
Fournier E., Repelin J. (1901) - Recherche sur le préhistorique de la Basse-Provence, Annales de la faculté des sciences de Marseille, 11, 9, p. 165-228.

GAGNIÈRE S. (1954) - Informations archéologiques : XII circonscription, Gallia Préhistoire, 12, 1, p. 115-127.

Gagnière S., Granier J. (1963) - Les stèles anthropomorphes du musée Calvet d'Avignon, Gallia Préhistoire, 6, p. 31-62.

GAGnière S., Granier J. (1967) - Nouvelles stèles anthropomorphes chalcolithiques de la vallée de la Durance, Bulletin de la Société préhistorique française, 64, 3, p. 699-706.

Gagnière S., Granier J. (1976) - Catalogue raisonné des stèles anthropomorphes chalcolithiques du musée Calvet d'Avignon, Avignon, musée Calvet, 75 p.

GagniÈre S., Granier J. (1977-1978) - Nouvelle stèle anthropomorphe néolithique trouvée prés de Goult, Vaucluse, Mémoires de l'académie de Vaucluse, 10, p. 53-56.

Gallay A. (1995) - Les stèles antropomorphes du site mégalithique du Petit-Chasseur à Sion (Valais, Suisse), in S. Casini, R. C. De Marinis et A. Pedrotti (dir.), Statue-stele e massi incisi nell'Europa dell'Età del Rame, Bergame, Civico museo archeologico (Notizie archeologiche bergomensi, 3) p. 167-194.

GÉRIN-RICARD H. DE (1899) - Statistiques préhistoriques et protohistoriques des Bouches-du-Rhône, du Var et des Basses-Alpes, Marseille, Typographie et lithographie Barlatier, $34 \mathrm{p}$.

GÉRIN-RICARD H. DE (1910) - Les stèles énigmatiques de Trets et d'Orgon, Mémoire de l'académie de Vaucluse (2 ${ }^{e}$ série), 10, p. 85-89.

Gérin-Ricard H. de, Arnaud D’agnel G. (1907) - Les antiquités de la vallée de l'Arc, Aix-en-Provence, B. Niel (Publication de la Société d'études provençales), 334 p.

Gernigon K. (2014) - Le Chasséen entre temps et espace : vingt ans de périodisations des assemblages céramiques et le retour de l'identité chasséenne, in I. Sénépart, F. Leandri, J. Cauliez et al. (dir.), Chronologie de la Préhistoire récente dans le Sud de la France : acquis 1992-2012, actualité de la recherche, actes des $10^{\text {es }}$ Rencontres méridionales de Préhistoire récente (Porticcio, 18-20 octobre 2012), Toulouse, Archives d'écologie préhistorique, p. 37-61.

Goguel J. (1966) - Carte géologique de la France au 1/50000, feuille Cavaillon, XXXI-42, Orléans, BRGM, Service géologique national.

Gotoshia S. V., Gotoshia L. V. (2008) - Laser Raman and Resonance Raman Spectroscopies of Natural Semiconductor Mineral Cinnabar, $\alpha-\mathrm{HgS}$, from Various Mines, Journal of Physics D: Applied Physics, 41, 11, p. 115406.

Grau Bitterli M.-H., Fierz-Dayer E. (2011) - Bevay/ Treytel-À Sugiez : histoire d'un complexe mégalithique néolithique, témoins d'habitats du Campaniforme et du Bronze ancien, plateau de Bevay 6, Neuchâtel, musée cantonal d'Archéologie (Archéologie neuchâteloise, 47), 373 p.

Guilaine J. (1998) - Au temps des dolmens. Mégalithes et vie quotidienne en France méditerranéenne, il y a 5000 ans, Toulouse, Privat, 167 p.

GuILAINE J. (2003) - Stèles anthropomorphes, statues et sociétés de la Préhistoire, cours du Collège de France, Paris, 
Collège de France, http://www.collège-de-France.fr-mediains_pro-UPL14515_JGuilaine_cours02_03 [en ligne].

Gutherz X., JaLlot L. (1987) - Statue-menhir et habitat néolithique final de Montaïon (Sanilhac-et-Sagriès, Gard), in Fédération archéologique de l'Hérault (dir.), Actes des journées d'étude des statues-menhirs : Saint-Pons-de-Thomières, mai 1984, Saint-Pons-de-Thomières, Fédération des usagers du parc naturel régional du Haut-Languedoc, p. 15-36.

Gutherz X., Jallot L. (1999) - Approche géoculturelle des pays fontbuxiens, in Vaquer J. (dir.), Le Néolithique $d u$ Nord-ouest méditerranéen, actes du XXIV Congrès préhistorique de France (Carcasonne, 26-30 septembre 1994) Paris, Société préhistorique française, vol. 2, p. 161-174.

Gutherz X., Jallot L., Garnier N. (1998) - Le monument de Courion (Collias, Gard) et les statues-menhirs de l'Uzège méridionale, in G. Rodriguez (dir.), Actes $d u 2^{e}$ Colloque international sur la statuaire mégalithique, Saint-Pons-deThomières du 10 au 14 septembre 1997, Lattes, Fédération archéologique de l'Hérault (Archéologie en Languedoc, 22), p. 119-134.

Hameau P. (1998) - Saint-Saturnin-lès-Apt : Font-Jouval, Baume Peinte, Bilan scientifique. Région Provence-AlpesCôte d'Azur 1997, direction régionale des Affaires culturelles de la région Provence-Alpes-Côte d'Azur, Aix-enProvence, p. 146-147.

Hameau P. (1999) - Gordes et Joucas, falaise de la Baume Brune, gorge de la Véroncle, Bilan scientifique. Région Provence-Alpes-Côte d'Azur 1998, direction régionale des Affaires culturelles de la région Provence-Alpes-Côte d'Azur, Aix-en-Provence, p. 164-165.

Hameau P. (2004) - Les peintures schématiques de la Combe de Fontjouval (Saint-Saturnin-lès-Apt, Vaucluse), in H. Dartevelle (dir.), Auvergne et Midi. Actualité de la recherche, actes des $5^{\text {es }}$ Rencontres méridionales de Préhistoire récente (ClermontFerrand, 8-9 novembre 2002), Cressensac, Préhistoire du SudOuest, (Supplément à Préhistoire du Sud-Ouest, 9) p. 457-466.

Hameau P., Cruz V., Laval E., Menu M., Vignaud C. (2001) Analyse de la peinture de quelques sites postglaciaires du SudEst de la France, L'Anthropologie (Paris), 105, 4, p. 611-626.

Hameau P., Menu M., Pomies M.-P., Walter P. (1995) - Les peintures schématiques postglaciaires du Sud-Est de la France : analyses pigmentaires, Bulletin de la Société préhistorique française, 92, 3, p. 353-362.

HASLER A. (1998) - Les stèles de la nécropole tumulaire néolithique de Château-Blanc (Ventabren, Bouches-du-Rhône), in G. Rodriguez (dir.), Actes $d u 2^{e}$ Colloque international sur la statuaire mégalithique, Saint-Pons-de-Thomières du 10 au 14 septembre 1997, Lattes, Fédération archéologique de l'Hérault (Archéologie en Languedoc, 22), p. 105-112.

Hasler A. (2014) - Une station du Néolithique final, une stèle anthropomorphe, in N. Nin (dir.), Aix en Archéologie : 25 ans de découvertes, [Gand], Snoeck, p. 65-68.

Hasler A., Chevillot P., Collet H., Durand C., Renault S., Richier A. (1998) - La nécropole tumulaire néolithique de Château Blanc (Ventabren, Bouches-duRhône), in A. D'Anna et D. Binder (dir.), Production et identité culturelle, actes de $2^{\text {es }}$ Rencontres méridionales de Préhistoire récente (Arles, 8-9 novembre 1996), Antibes, APDCA, p. 403-414.
Hasler A., Collet H., Durand C., Chevillot P., Renault S., RichieR A. (2002) - Fiche no 22. Ventabren, Château Blanc. Une nécropole tumulaire néolithique, in Archéologie du TGV Méditerranée : fiches de synthèse, 1. La préhistoire, Lattes, Association pour la recherche archéologique en Languedoc oriental (Monographies d'archéologie méditerranéenne, 8), p. 227-238.

HAÜSLER A. (1966) - Anthropomorphe Stelen des Eneolithikums im Nordpontischen Raum, Wissenschaftliche Zeitschrift der Martin-Luther-Universität, 15, p. 29-73.

Hermet F. (1898) - Statues-menhirs de l'Aveyron et du Tarn, Bulletin archéologique du Comité des travaux historiques et scientifiques, 1898, p. 500-536.

Hochleitner R. (1984) - Atlas des roches et minéraux, Paris, Fernand Nathan, 239 p.

JALLOT L. (1987) - Nouvelles données sur les statues-menhirs du Languedoc oriental, in Fédération archéologique de l'Hérault (dir.), Actes des journées d'étude des statuesmenhirs, Saint-Pons-de-Thomières, 5-6 mai 1984, SaintPons-de-Thomières, Fédération des usagers du parc naturel régional du Haut-Languedoc, p. 37-55.

JALLOT L. (1998) - Enquête typologique et chronologique sur les menhirs anthropomorphes : études de cas dans le Sud de la France, l'Ouest, l'arc Alpin et la Bourgogne, in G. Rodriguez (dir.), Actes du $2^{e}$ Colloque international sur la statuaire mégalithique, Saint-Pons-de-Thomières du 10 au 14 septembre 1997, Lattes, Fédération archéologique de l'Hérault (Archéologie en Languedoc, 22), p. 317-350

JALLOT L. (2003) - La culture matérielle du site des Vautes, La stèle de l'Aven 2, le menhir des Vautes et les stèles non figuratives du Languedoc, in J. Guilaine et G. Escallon (dir.), Les Vautes (Saint-Gély-du-Fesc, Hérault) et le Néolithique final du Languedoc oriental, Toulouse, Centre d'anthropologie, École des hautes études en sciences sociales et INRAP, p. 131-136

JALLOT L. (2011) - Frontières, stabilités, emprunts et dynamique géoculturelle en Languedoc méditerranéen au Néolithique final (3400-2300 av. J.-C.), in I. Sénépart, T. Perrin, E. Thirault et al. (dir.), Marges, frontières et transgressions : Actualité de la recherche, actes des $8^{\text {es }}$ Rencontres méridionales de Préhistoire récente (Marseille, 7-8 novembre 2008), Toulouse, Archives d'écologie préhistorique, p. 87-119.

LANDAU J. (1977) - Les représentations anthropomorphes mégalithiques de la région méditerranéenne (III ${ }^{e}$ au $I^{e r}$ millénaire), Paris, CNRS (Centre de recherches archéologiques, Publications de l'URA no 7, Mémoires 1), $130 \mathrm{p}$.

Le Quellec J.-L., Fauvelle-Aymar F.-X., Bon F. (2009) Essai de reconstitution d'un panneau rupestre : la scène de bataille de "Christol Cave », Afrique-du-Sud, L'Anthropologie (Paris), 113, 5 (« Représentations préhistoriques : images du sens », sous la direction scientifique de Denis Vialou), p. 820-838.

Le Quellec J.-L., Harman J., Defrasne C., DuquesNOY F. (2013) - DStretch ${ }^{\circledR}$ et l'amélioration des images numériques: applications à l'archéologie des images rupestres, Cahiers de l'AARS, 16, p. 177-198.

LÉA V. (2004) - Les industries lithiques du Chasséen en Languedoc oriental : caractérisation par l'analyse technologique, Oxford, J. and E. Hedges (British archaeological Reports, International Series 1232), v 215 p. 
LÉa V., Gassin B., Linton J. (2009) - Quelles armatures de projectiles pour le Midi méditerranéen et ses marges du milieu du Ve millénaire au milieu du IV e millénaire?, Gallia Préhistoire, 51, p. 155-177.

Léa V., Georjon C., Lepère C., Sénépart I., Thirault E., Avec la Collaboration De Carry A., Grenet M., Gassin B., Bouby L., Devalque C., Garaix L. (2004) - Chasséen vauclusien, qui es-tu?, in J. Buisson-Catil, A. Guilcher, C. Hussy et al. (dir.), Vaucluse préhistorique : le territoire, les hommes, les cultures et les sites, Le Pontet, Barthélémy, p. 163-200.

LEPÈRE C. (2012) - Chronologie des productions céramiques et dynamiques culturelles du Chasséen de Provence, Bulletin de la Société préhistorique française, 109, 3, p. 513-545.

López Melción J. B., Moya Garra A., Escala Abad O., Nieto Espinet A. (2009) - La cista tumulària amb esteles esculpides dels Reguers de Seró (Artesa de Segre, Leida), una aportacio insolita dins de l'art megalitic peninsular i europeu, Tribuna d'arqueologia, 2008-2009, p. 87-125.

Lugliè C., Le Bourdonnec F.-X., Poupeau G. (2014) Caratterizzazione elementare e provenienza delle ossidiane mediante analisi non distruttiva PIXE e EDXRF, in M. Venturino Gambari (dir.), La memoria del passato. Castello di Annone tra archeologia e storia, Alessandria, LineLab edizioni, p. 333-336.

Lugliè C., Le Bourdonnec F.-X., Poupeau G., Bohn M., Meloni S., Oddone M., Tanda G. (2006) - A Map of the Monte Arci (Sardinia Island, Western Mediterranean) Obsidian Primary to Secondary Sources: Implications for Neolithic Provenance Studies, Comptes Rendus Palevol, 5, 8, p. 995-1003.

MARCHESI H. (1990) - L'occupation de la moyenne vallée du Calavon du Néolithique à la fin de l'Antiquité, Avignon, service d'archéologie de Vaucluse (Notices d'archéologie vauclusienne, 1), $71 \mathrm{p}$.

Mezzena F. (1998) - Le stele antropomorfe nell'area megalitica di Aosta $=$ Les stèles anthropomorphes de l'aire mégalithique d'Aoste, in F. Ambrosio (dir.), Dei di pietra: la grande statuaria antropomorfa nell'Europa del III millennio a.C. = Dieux de pierre : la grande statuaire anthropomorphe en Europe au III millénaire avant J.-C., Milan, Skira, p. 90-127.

Müller D. W. (1997) - Ornamente, Symbole, Bilder - zum megalitischen Totenbrauchtum in Mitteldeutschland, in J. L'Helgouach, C.-T. Le Roux et J. Lecornec (dir.), Art et symboles du mégalithisme européen, actes du $2^{\mathrm{e}}$ Colloque international sur l'art mégalithique (Nantes, juin 1995), Rennes, Pôle éditorial archéologique de l'Ouest (Supplément à la Revue archéologique de l'Ouest, 8), p. 163-178.

Muséum national D'histoire naturelle (2015) - Cinabre, Paris, Muséum national d'histoire naturelle, galerie de minéralogie et de géologie, http://www.galeriedemineralogieetgeologie.fr/fr/explorez/150-especes-minerales/c-calcite/cinabre [en ligne].

Остовоn F. C. Е. (1931) - Enquête sur les figurations Néo- et Enéolithique. Statues-menhirs, stèles gravées, dalles sculptées, Revue anthropologique, 41, p. 297-576.

Pellissier M. (2010) - Le site chasséen « les Terres Longues » à Trets (Bouches-du-Rhône), in X. Delestre et H. Marchesi (dir.), Archéologie des rivages méditerranéens : 50 ans de recherche, actes du colloque (Arles, 28-30 octobre 2009), Paris, Errance, p. 329-335.

Philippon A., dir. (2002) - Statues-menhirs : des énigmes de pierre venues du fond des âges. Rodez, Éd. du Rouergue, 271 p.

Poggiani Keller R. (2013) - Le sanctuaire mégalithique d'Ossimo-Pat (Valcamonica, Brescia, Italie), in M. A. Borrello (dir.), Les hommes préhistoriques et les Alpes, Oxford, Archaeopress (British archaeological Reports, International Series 2476), p. 219-226.

PotTIER R. (1878) - Stations néolithiques de Gargas, Vaucluse, Matériaux pour l'histoire primitive et naturelle de l'homme, 9, p. 183-185.

Poupeau G., Lugliè C., D'Anna A., Carter T., Le Bourdonnec F.-X., Bellot-Gurlet L., Bressy C. (2010) - Circulation et origine de l'obsidienne préhistorique en Méditerranée : un bilan de cinquante années de recherches, in X. Delestre et H. Marchesi (dir.), Archéologie des rivages méditerranéens : 50 ans de recherche, actes du colloque (Arles, 28-30 octobre 2009), Paris, Errance, p. 183-191.

Rodriguez G. (1998) - L'évolution de la statuaire mégalithique en haut Languedoc et ses différences avec la Rouergate, in G. Rodriguez (dir.), Actes du $2^{e}$ Colloque international sur la statuaire mégalithique, Saint-Pons-de-Thomières du 10 au 14 septembre 1997, Lattes, Fédération archéologique de 1'Hérault (Archéologie en Languedoc, 22), p. 167-181.

Rouquette D. (1998) - La stèle gravée du Truc de Martin à Viols-le-Fort (Hérault), in G. Rodriguez (dir.), Actes $d u$ $2^{e}$ Colloque international sur la statuaire mégalithique, Saint-Pons-de-Thomières du 10 au 14 septembre 1997, Lattes, Fédération archéologique de l'Hérault (Archéologie en Languedoc, 22), p. 141-144.

Saintot S., Brochier J. L. (2002) - Fiche no 4. L'occupation chasséenne du site de Chabrillan, La Prairie (Drôme), in Archéologie du TGV Méditerranée : fiches de synthèse, 1. La préhistoire, Lattes, Association pour la recherche archéologique en Languedoc oriental, (Monographies d'archéologie méditerranéenne, 8), p. 43-56.

Sautel J., Gagnière S., Germand L. (1931) - Essai historique sur le département de Vaucluse : la Préhistoire, Lyon, A. Rey, $101 \mathrm{p}$.

SAuzade G. (1983) - Les Sépultures du Vaucluse, du Néolithique à l'âge du Bronze, Paris, Éd. du laboratoire de paléontologie humaine et de Préhistoire, Institut de paléontologie humaine (Études quaternaires, 6), $251 \mathrm{p}$.

Sauzade G., avec la collaboration de Castan M. (1987) Découverte au village de Beaucet (Vaucluse) d'une nouvelle stèle anthropomorphe à chevrons du Néolithique, Bulletin de la Société préhistorique française, 84, 6, p. 172-176.

SAuzAde G. (2012) - Offrandes, mobiliers et intentions perceptibles dans les sépultures provençales entre 3500 et 1800 ans av. J.-C., in M. Sohn et J. Vaquer (dir.), Sépultures collectives et mobiliers funéraires de la fin du Néolithique en Europe occidentale, actes de la table ronde « La fin du Néolithique en Europe de l'Ouest. Valeurs sociales et identitaires des dotations funéraires (3500-2000 av. n. è.) » (Carcassonne, 26-28 septembre 2008), Toulouse, Archives d'écologie préhistorique, p. 177-212. 
Sauzade G., Buisson-Catil J., Bizot B. (2003) - Le dolmen de l'Ubac et son environnement immédiat (Goult, Vaucluse), in J. Gascó, X. Gutherz et P.-A. de Labriffe (dir.), Temps et espaces culturels : du VIe au II millénaire en France du Sud, actes des $4^{\text {es }}$ Rencontres méridionales de Préhistoire récente (Nimes, 28-29 octobre 2000), Lattes, ADAL (Monographies d'archéologie méditerranéenne, 15), p. 335-346.

SAuzade G., Cerclier O. (2014) - Un nouveau style de stèle à décor de chevrons : Le Beaucet 2 (Vaucluse) et Miouvin (Istres, Bouches-du-Rhône). Précisions sur les contextes et la chronologie des stèles provençales, Bulletin archéologique de Provence, 36, p. 13-30.

Schmiтt A. (2015) - Pratiques mortuaires en fosse au Néolithique moyen dans le Midi de la France : caractérisations et éclairages interprétatifs, L'Anthropologie (Paris), 119, 1, p. 1-37.

Syndicat intercommunal de la mine de Cap Garonne, (2015) - La mine de Cap Garonne, Le Pradet, syndicat intercommunal de la mine de Cap Garonne, http://www. mine-capgaronne.fr/ [en ligne].

Thirault É. (2006) - Un point sur la question des billes chasséennes, in L. Baray (dir.), Artisanats, sociétés et civilisations : hommage à J.-P. Thévenot, actes du colloque (Sens, 2-3 avril 2003), Dijon, Revue archéologique de l'Est (supplément à la Revue archéologique de l'Est, 24), p. 145-161.

Түкот R. H. (1997) - Characterization of the Monte Arci (Sardinia) Obsidian Sources, Journal of Archaeological Science, 24,5 , p. $467-479$

Van Willigen S., D’Anna A., Renault S., Sargiano J.-P. (2012) - Le Sud-Est de la France entre 4400 et 3400 avant notre ère. Sériation céramique et outillage lithique, Préhistoires méditerranéennes, 2, http://pm.revues.org/index601. $\mathrm{html}$ [en ligne].

VAquer J. (1991) - Aspects du Chasséen en Languedoc occidental : habitat et culture matérielle, in A. Beeching, D. Binder, J.-C. Blanchet et al. (dir.), Identité du Chasséen, actes du colloque international (Nemours, 1989), Nemours, APRAIF (Mémoire du musée de Préhistoire d'Île-deFrance, 4) p. 27-37.

VAQUER J. (2007) - Le rôle de la zone nord-tyrrhénienne dans la diffusion de l'obsidienne en Méditerranée nord-occidentale au Néolithique, in A. D’Anna, J. Cesari, L. Ogel et al. (dir.), Corse et Sardaigne préhistoriques : relations et échanges dans le contexte méditerranéen, Paris, CTHS (Documents préhistoriques, 22) p. 99-119.

Voruz J.-L. (1990) - Litholâtrie néolithique : les statuesmenhirs de Suisse romande, in R. Joussaume (dir.), Mégalithisme et société, actes de la table ronde (Les Sablesd'Olonne, 1987), La Roche-sur-Yon, Groupe vendéen d'études préhistoriques, p. 187-207.

Voruz J.-L. (1992) - Hommes et dieux du Néolithique. Les statues-menhirs d'Yverdon, Jahrbuch der Schweizerischen Gesellschaft für Ur- und Frühgeschichte, 75, p. 37-64.
Walter P., Louboutin C., Hasler A. (1997) - Les stèles anthropomorphes de la Bastidonne, Trets (Bouches-duRhône) et l'usage de la couleur sur les stèles provençales de la fin du Néolithique, Antiquités nationales, 29, p. 27-33.

WÜTrICH S. (2003) - Saint-Aubin, Derrière la Croix. Un complexe mégalithique durant le Néolithique moyen et final, Hauterive, Neuchâtel, service et musée cantonal d'archéologie de Neuchâtel (Archéologie neuchâteloise, 29), 363 p.

\section{André D'Anna \\ Jean-Louis GuENDON Adrien RegGio Stéphane RenaulT}

UMR 7269 « Laboratoire méditerranéen de Préhistoire Europe Afrique », Aix-Marseille université, CNRS, ministère de la Culture,

Maison méditerranéenne des sciences de l'homme, 5 rue du Château de l'Horloge BP 647 - F-13094 Aix-en-Provence Cedex 2 danna@mmsh.univ-aix.fr adrien.reggio@gmail.com renault@mmsh.univ-aix.fr

Christiane Bosansky 2, campagne Beyssan, F-84400 Apt

Ludovic Bellot-GuRLET

UMR 8233 «MONARIS, de la molécule aux nano-objets : réactivité, interactions et spectroscopies $"$, université Pierre-et-Marie-Curie-Paris 6, CNRS, 4, pl. Jussieu, F-75252 Paris Cedex 05, ludovic.bellot-gurlet@upmc.fr

François Xavier Le BouRdonNEC UMR 5060 « IRAMAT-CRP2A, Institut de recherche sur les archéomatériaux, centre de recherche en physique appliquée à l'archéologie », université Bordeaux Montaigne, CNRS, Maison de l'archéologie, Esplanade des Antilles, F-33607 Pessac Cedex Francois-Xavier.Le-Bourdonnec@u-bordeauxmontaigne.fr 